\title{
FREEMARTINS IN CATTLE: THE FIRST STEPS OF SEXUAL ORGANOGENESIS
}

\author{
A. JOST, B. VIGIER AND J. PREPIN \\ Laboratory of Comparative Physiology, Faculty of Sciences, University Paris VI, \\ 9 Quai Saint-Bernard, Paris 5, France
}

(Received 1st April 1971)

Summary. Multiple pregnancies were obtained in cows treated with gonadotrophic hormones and the fetuses were removed 39 to 62 days after mating. This covers the period of sexual organogenesis in males from the initial differentiation of the testes to the final establishment of most male structures. Twenty-nine pregnancies resulting in heterosexual fetuses with fused chorions and vascular anastomoses provided forty-eight presumptive freemartins. The genital tract was studied histologically and gonadal size was determined by the paper weight method.

No difference was observed between control females and presumptive freemartins up to 48 days. Thereafter, growth of the presumptive ovaries practically stopped in freemartins and the diameter of the upper part of their Müllerian ducts decreased, as in males. Contrary to older views, the first freemartin effects occurred at the same stage (49 to 52 days) in all the presumptive freemartins.

Ovarian and Müllerian inhibition begins simultaneously and a significant correlation between the degree of inhibition of both structures was obtained. The penis, scrotum and prostate were absent in all freemartins until Day 60. In only one case was the ano-genital distance slightly increased. A delayed appearance of seminal vesicles was found in three out of seven freemartins at 61 and 62 days.

There was a notable absence of seminiferous tubules in the gonads of all these freemartins. It is suggested that if these tubules are present after birth it is because they have developed later.

The factors responsible for the freemartin effect still remain to be discovered. Ovarian and Müllerian inhibitions occur during the period of time when the external genitalia of males become masculinized. This suggests that a factor of male origin which inhibits both the ovaries and the Müllerian ducts and which is distinct from the masculinizing hormone could be involved. Other possibilities are also discussed.

\section{INTRODUCTION}

The hormonal explanation of the origin of freemartins in cattle has dominated the theory behind experimental research on sex differentiation for more than 
half a century (Keller \& Tandler, 1916; Lillie, 1916, 1917). Freemartins studied after birth are characterized by several very variable features: the ovaries are reduced in size and sterile, and they frequently contain sterile seminiferous tubules; the derivatives of the Müllerian ducts are more or less completely absent; the presence of seminal vesicles is common (Lillie, 1923; Bissonnette, 1924) although a prostate and masculinized external genitalia are of rare occurrence. In the hormonal theory of freemartinism, it is assumed that

\section{TABLE 1}

SURVEY OF THE SEXUAL DIFFERENTIATION OF THE GALF FETUS, ACCORDING TO OUR OWN OBSERVATIONS AND TO THE LITERATURE (MODIFIED FROM JOST, 1966)

\begin{tabular}{|c|c|c|c|}
\hline $\begin{array}{c}\text { Age } \\
(\text { days })\end{array}$ & $\begin{array}{l}\text { Crown-rump } \\
\text { length } \\
(\mathrm{mm})^{*}\end{array}$ & Males & Females \\
\hline 39 to 40 & & Early testicular organogenesis $\dagger$ & Undifferentiated gonadal primordium \\
\hline 42 to 43 & 24 to 26 & Definite albuginea & \\
\hline 45 & 29 & Testicular interstitial cells & \\
\hline 47 & 32 & First flexure of penile urethra & \\
\hline 48 to 49 & 34 to 36 & $\begin{array}{l}\text { Anogenital distance definitely } \\
\text { increased } \\
\text { Growing Müllerian }\end{array}$ & $\begin{array}{l}\text { Beginning of prolonged and slow } \\
\text { thickening of the superficial ovarian } \\
\text { layers } \\
\text { ducts in both sexes }\end{array}$ \\
\hline 50 to 52 & 38 to 42 & $\begin{array}{l}\text { Upper Müllerian ducts: reduction } \\
\text { in diameter }\end{array}$ & \\
\hline 56 & 53 & $\begin{array}{l}\text { Prostatic buds and seminal vesicles } \\
\text { appear } \\
\text { Male urogenital connections }\end{array}$ & \\
\hline 58 to 60 & 59 to 66 & $\begin{array}{l}\text { Müllerian ducts: anterior part } \\
\text { disappears } \\
\text { Penis opens under the umbilicus. } \\
\text { Balanopreputial fold in organization } \\
\text { Scrotum develops }\end{array}$ & Uterus increases in diameter \\
\hline 70 & 115 & $\begin{array}{l}\text { Müllerian ducts absent or regressing } \\
\text { Formation of epididymis }\end{array}$ & Retrogression of Wolffian ducts begins \\
\hline 75 & - & & First premeiotic figures $\ddagger$ \\
\hline
\end{tabular}

* Length in mm calculated from a regression line (crown-rump length/age) established for our material between the ages of 42 and 70 days.

† Stage of $25 \mathrm{~mm}$ (Lillie, 1923); Day 39 according to Krehbiel (1963) and Erickson (1966).

$\ddagger$ According to Erickson (1966).

a testicular hormone which governs the differentiation of the genital structures in the male fetus is transferred to the freemartin through placental vascular anastomoses and influences the development of the genital tract. More recently, it has been suggested that transfer of cells rather than an exchange of hormones could produce the freemartin effect, though the nature and the mode of action of these cells has not been specified (Fechheimer, Herschler \& Gilmore, 1963; Goodfellow, Strong \& Stewart, 1965; Kanagawa, Kawata \& Ishikawa, 1965; Herschler \& Fechheimer, 1967).

The causative factors of the freemartin effect still have to be discovered and precise developmental studies can be expected to shed further light on the problem. Therefore, a series of freemartins were obtained in superovulated 
cows and studied at known stages during the early differentiation of the genital tract (Table 1).

It is generally accepted that, in normal males, testes first become histologically recognizable on about Day 39 or 40 whereas, in females, the ovaries are slow in differentiating; the first primary follicles appear after the 12th week, heralding the differentiation of a true ovary (Thomson, 1942; Sakai, 1955; Erickson, 1966; Gropp \& Ohno, 1966). It must be emphasized that when the female

TABLE 2

DATA FROM THE LITERATURE GONGERNING EARLY FREEMARTINS

\begin{tabular}{|c|c|c|c|c|}
\hline $\begin{array}{c}\text { Crown-rump } \\
\text { length } \\
(\mathrm{mm})\end{array}$ & $\begin{array}{c}\text { Age } \\
\text { (days) }\end{array}$ & Presumptive ovaries & Müllerian ducts & Author $\ddagger$ \\
\hline \multirow{4}{*}{32} & $45^{*}$ & Normal & Normal & (1) \\
\hline & $47 \dagger$ & $\begin{array}{l}\text { Slightly reduced } \\
\text { Pflüger's cords present }\end{array}$ & Slightly reduced & (2) \\
\hline & $48^{*}$ & Approximately normal & Normal & (1) \\
\hline & $49 *$ & Approximately normal & Normal & (1) \\
\hline \multirow[t]{5}{*}{$37 \cdot 5$} & $50 \dagger$ & $\begin{array}{l}\text { Reduced size } \\
\text { No Pflüger's cords }\end{array}$ & Normal & (3) \\
\hline & $60^{*}$ & $\begin{array}{l}\text { Slightly reduced size } \\
\text { Pflüger's cords present }\end{array}$ & & (4) \\
\hline & $60^{*}$ & Pflüger's cords present & & (4) \\
\hline & $60^{*}$ & Stunted size & Inhibited & (1) \\
\hline & $61^{*}$ & Stunted size & Inhibited & (1) \\
\hline $\begin{array}{l}67.5 \text { and } 75 \\
\text { (littermates) }\end{array}$ & 60 to $62 \uparrow$ & Reduced size & $\begin{array}{l}\text { Less reduced than in } \\
\text { males }\end{array}$ & (5) \\
\hline 75 & $62 \dagger$ & $\begin{array}{l}\text { Very reduced } \\
\text { No Pflüger's cords }\end{array}$ & As in males & (6) \\
\hline 90 & $66 \dagger$ & $\begin{array}{l}\text { Slightly reduced } \\
\text { Pflüger's cords present }\end{array}$ & Reduced and interrupted & (7) \\
\hline 90 & $66 \dagger$ & $\begin{array}{l}\text { Reduced } \\
\text { No Pflüger's cords }\end{array}$ & Vestigial & (7) \\
\hline
\end{tabular}

* Exact postfertilization age.

$\uparrow$ Age evaluated according to the growth curve established for our material.

$\ddagger$ (1) Jost, Vigier \& Prepin (1966), quoted in Jost (1966); (2) Bissonnette (1928b); (3) Lillie (1923); (4) Rajakoski \& Hafez (1964); (5) Bissonnette (1928a); (6) Chapin (1917); (7) Bissonnette (1924).

fetal gonads are referred to as ovaries at an early stage of development, they are still largely undifferentiated.

In male fetuses, the differentiation of masculine characteristics of the genital tract proceeds rapidly between Days 47 and 60 . Successively, the male external genitalia differentiate, the Müllerian ducts regress and the seminal vesicles, prostate and scrotum appear. In females at this time, little differentiation occurs except for' the thickening of the superficial layer of the presumptive ovary ('Pflüger's cords' of early authors) around an undifferentiated inner cellular mass.

Information about the early stages of sex differentiation in freemartins is scanty (Table 2) and frequently imprecise as to developmental age since the 
material was usually collected at the slaughterhouse. Gestational age can be deduced from fetal size, but this gives only a rough indication. No freemartin effect was observed at 45 days in presumptive freemartins but, thereafter, inhibition of growth of the gonad and especially of its cortical layer are the most prominent features, as emphasized by Lillie $(1917,1923)$ and Bissonnette $(1928 \mathrm{a}, \mathrm{b})$. No seminiferous tubules have been observed in early freemartins, and this was true for our forty-six freemartins under 62 days of age (Jost, 1970); the ovarian 'medulla' remained as a mass of undifferentiated cells, as in normal females (Plate 3). The first gonadal alterations in freemartins only appear several days after the testes have been established in males and they do not include any sign of testicular development (Jost, 1966, 1970). Evidence based on early freemartins gives no clue as to whether the initial differentiation of the testes from the undifferentiated gonadal primordia is hormonally controlled, as was sometimes supposed (see reviews by Willier, 1939; Burns, 1961).

At the level of the genital tract, the most constant anomaly in young freemartin fetuses is the inhibition of the Müllerian ducts. Other male sex characters are infrequent in freemartins at similar stages. It should be emphasized that as a rule the external genitalia remain unaffected although in the male twin they become masculine at the stage when in freemartins the development of the ovaries and the Müllerian ducts is inhibited (Jost, Vigier \& Prepin, 1966, quoted in Jost, 1966).

The freemartins examined in the present paper were obtained during the 3 weeks following the sexual differentiation of the testes in order to study the early development of the condition. Histological details of the gonads will be reported in a future paper.

\section{MATERIAL AND METHODS}

\section{Collection of freemartins}

Apart from two spontaneous twin pregnancies, multiple pregnancies were produced experimentally by superovulation with gonadotrophic hormones, in collaboration with the members of the Physiological Station of the I.N.R.A. at Jouy en Josas, and according to their technique (Mauléon, Mariana, Benoit, Solari \& Chupin, 1970). This treatment consists of one intramuscular injection of 2000 to 3000 i.u. PMSG 4 days before expected day of oestrus, and one intravenous injection of 1500 or 2000 i.u. HCG on the day of oestrus.

The cows were slaughtered a variable number of days after insemination, and the uterus brought to the dissecting room within $10 \mathrm{~min}$. It was carefully opened longitudinally so as to preserve the fetal membranes, and the superficial chorionic blood vessels were studied and sometimes photographed (Plate 1). The presence of an anastomosis between the chorionic vessels was verified in cases of multiple pregnancy.

Each fetus was then dissected out, weighed or measured, and the abdominal cavity opened and the genital tract exposed. In many instances, liver tissue was cultured for chromosome studies. The entire fetus was then fixed in Bouin's fluid. The posterior part of the body with the genital tract in situ was serially sectioned at $7.5 \mathrm{~mm}$ for histological studies. Our material consisted of twenty- 


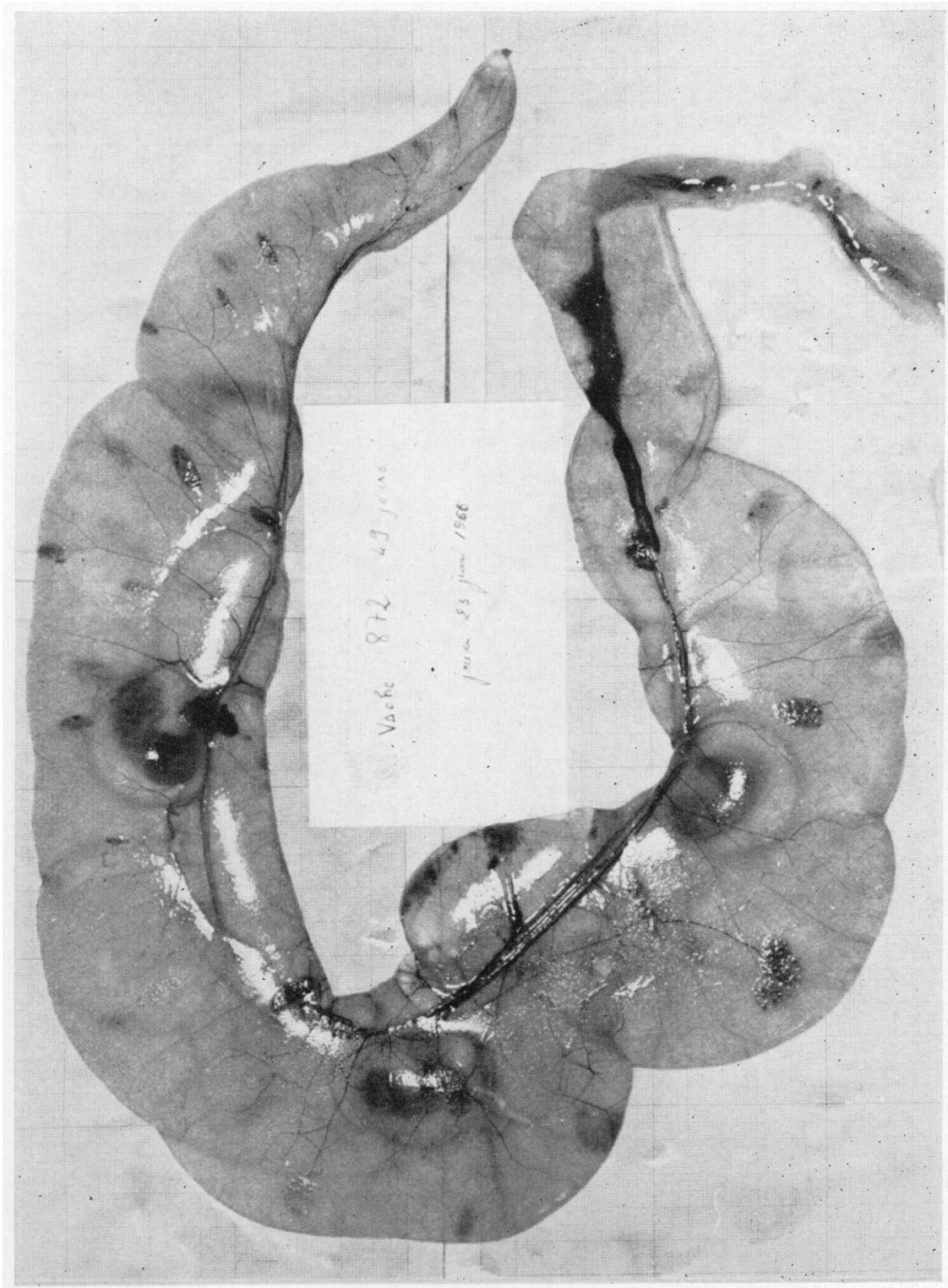

Fused chorio-allantois in a triplet set, 49 days after insemination (No. 872). The three fetuses contained in their individual amnions show through the chorio-allantois. Anastomoses of both large and small blood vessels are visible. The fetus in the middle was a female, the others were males. (Scale-5-cm large squares seen on the graduated paper). 


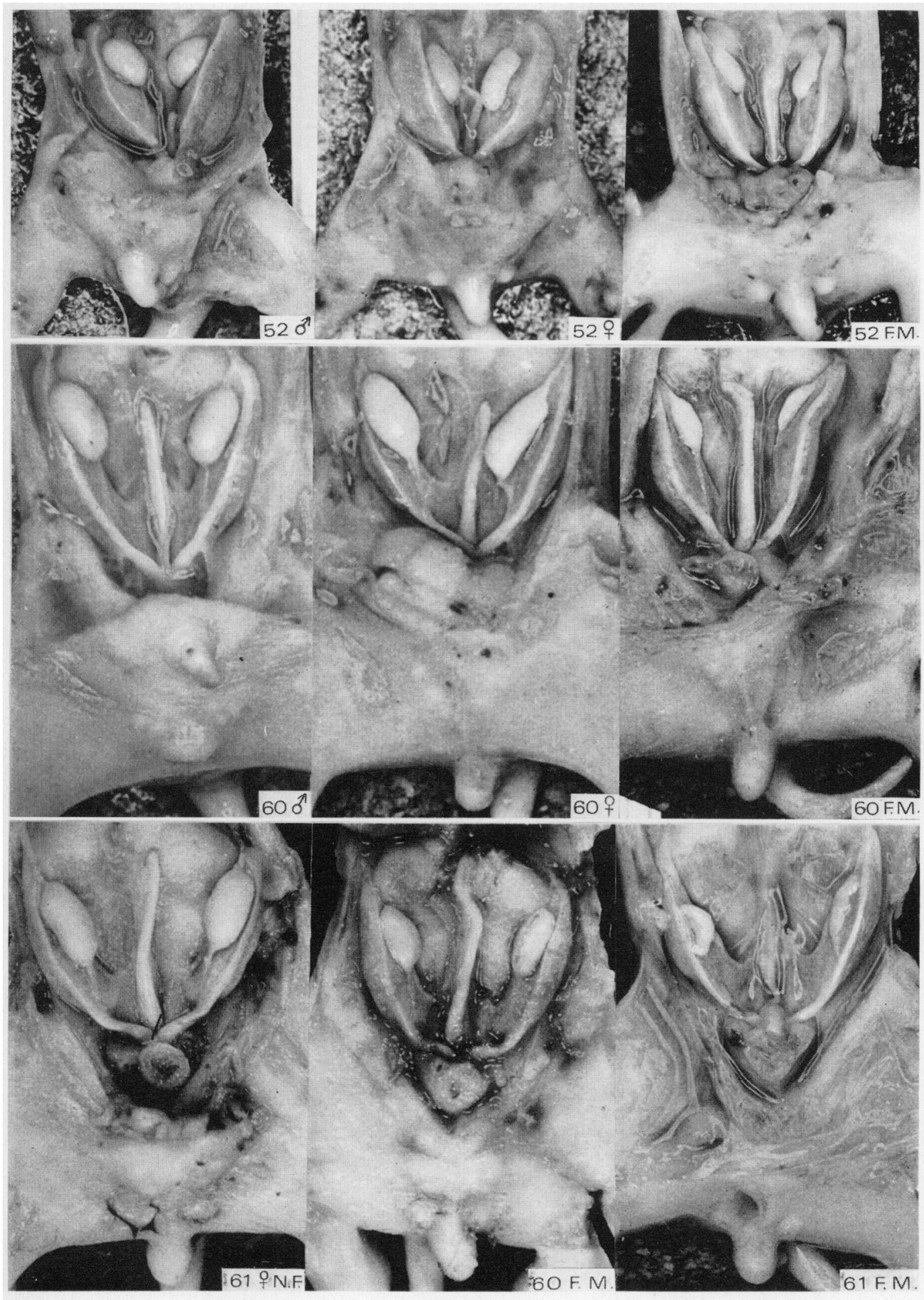



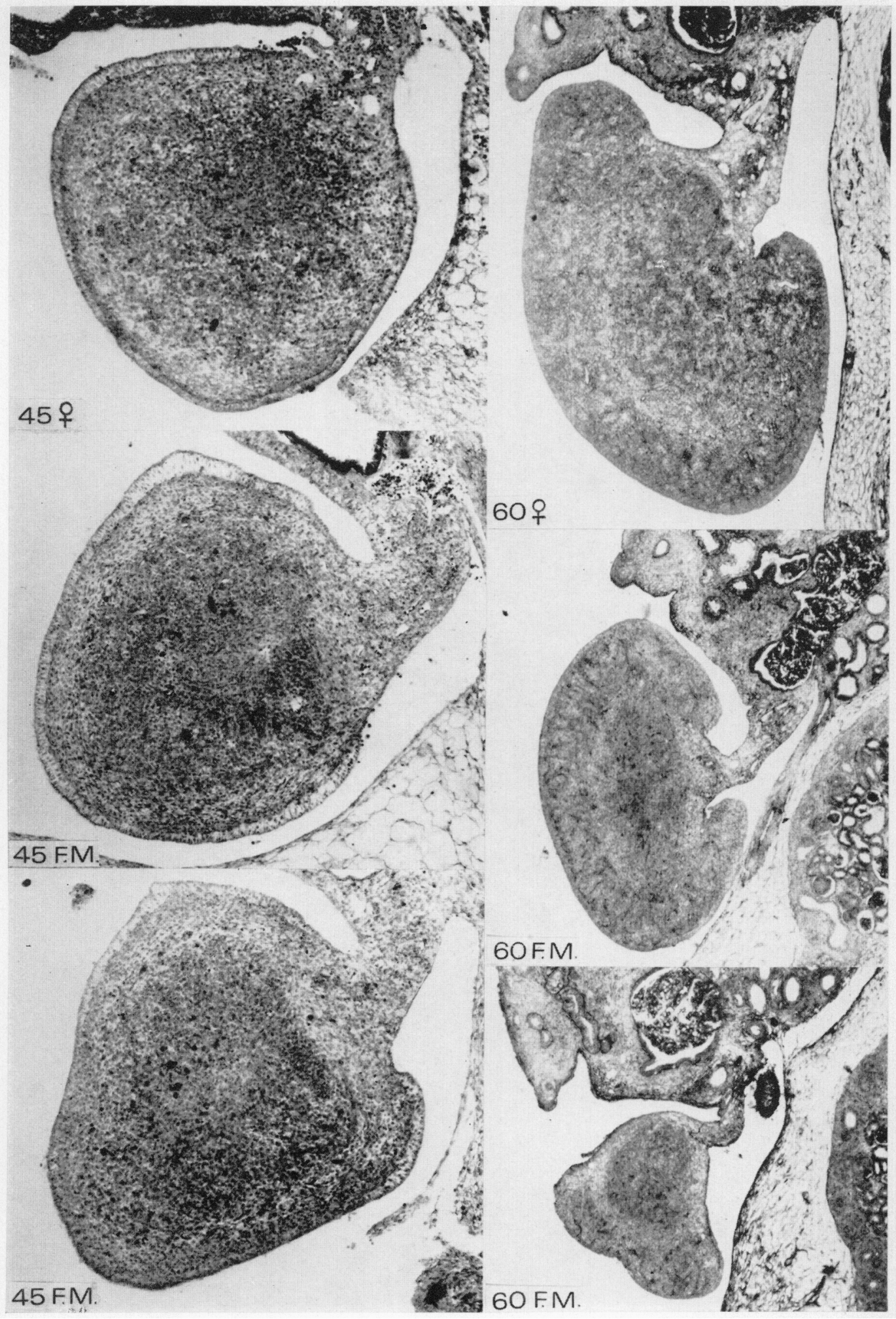


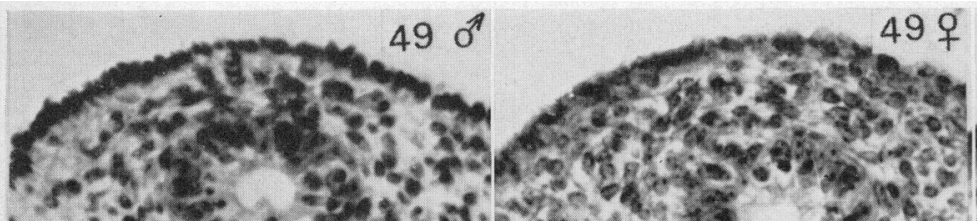
2.

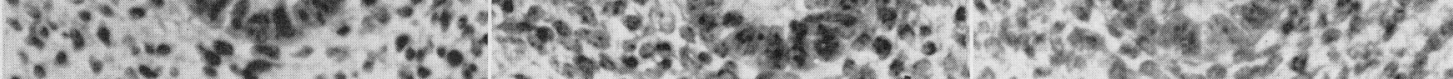

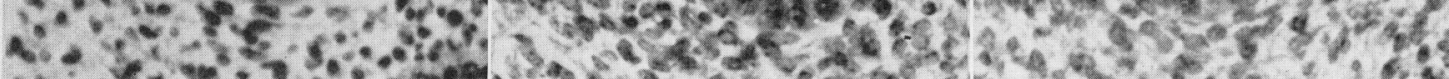

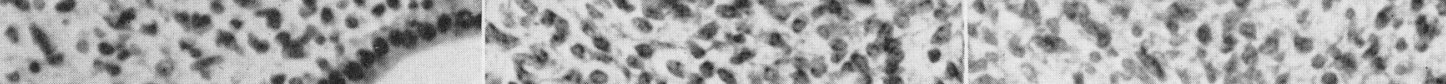

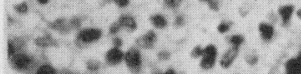
-

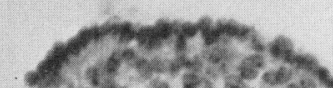

$520^{7}$

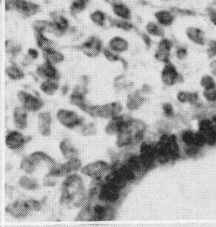

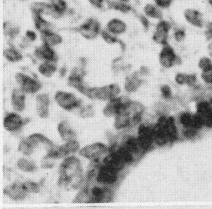
$x^{2}+\sin ^{2}$

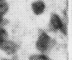

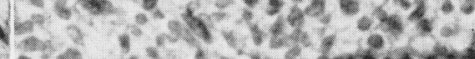

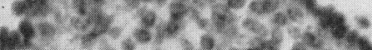
S.

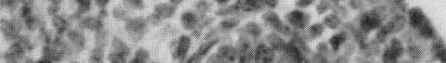
w arisente 8.

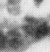
at

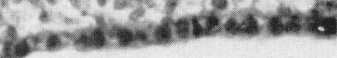

52 우

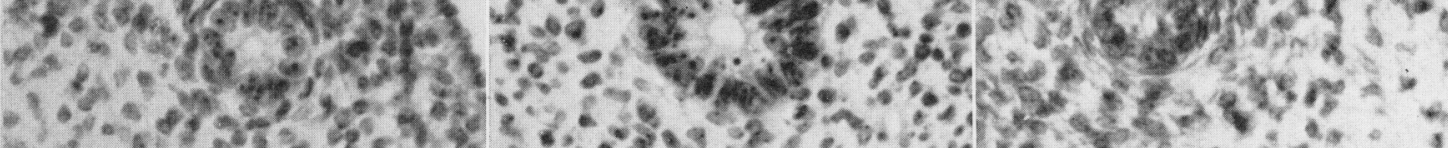

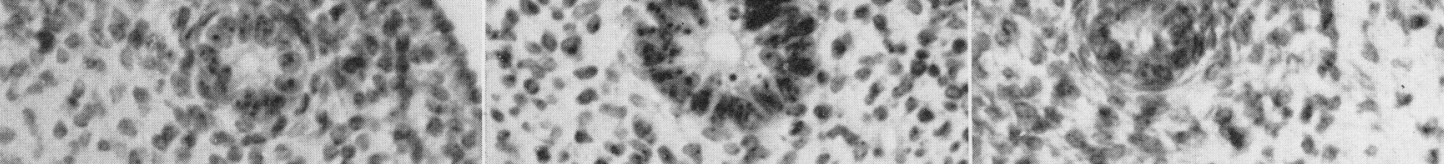

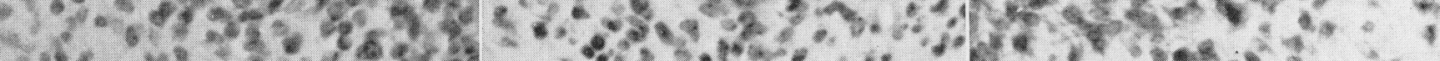

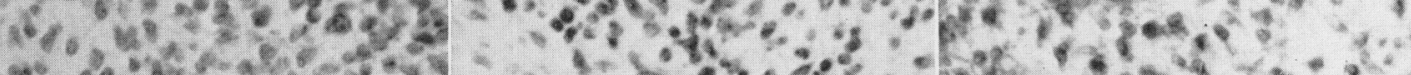

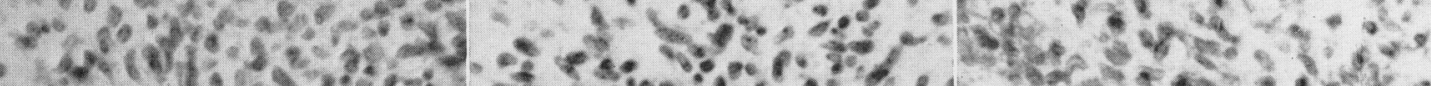

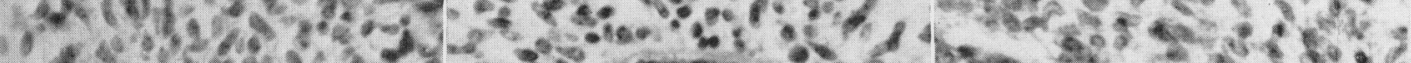

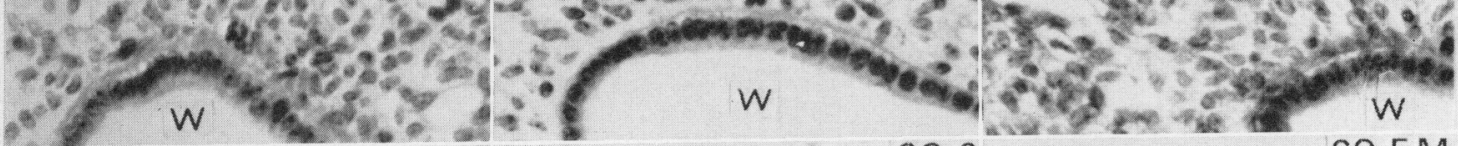

60 F.M.
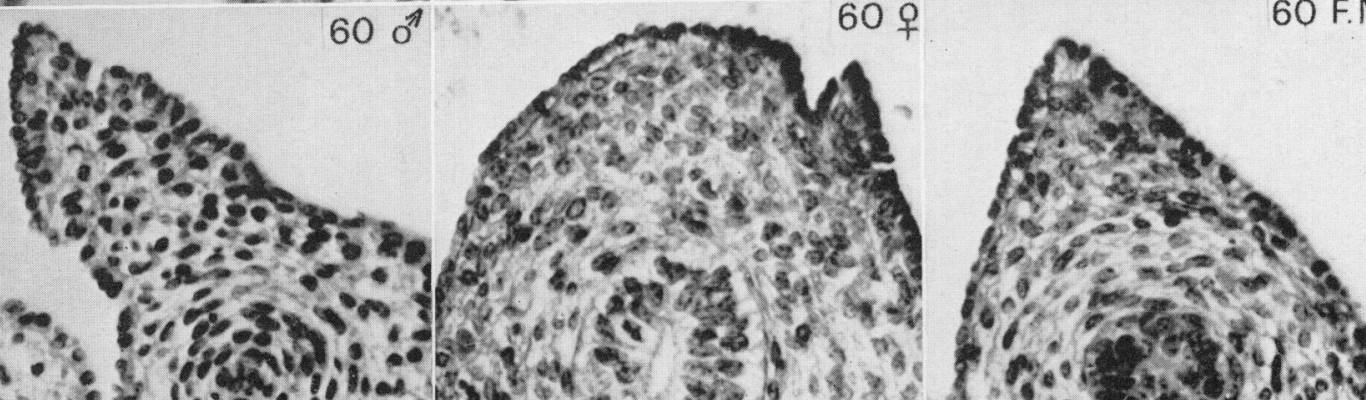

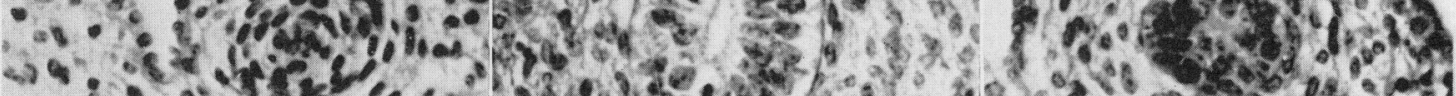

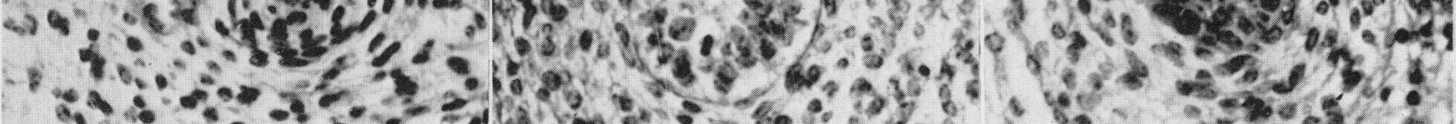

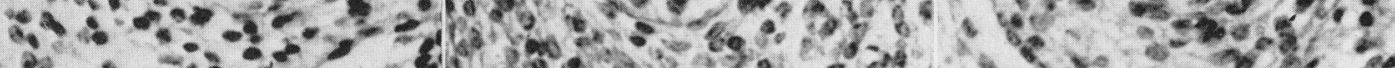

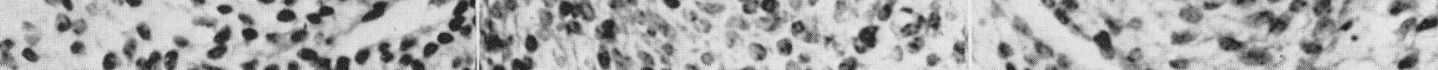

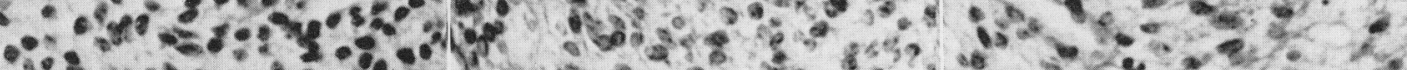
18 \&

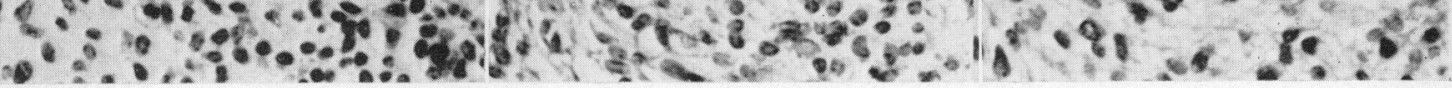


nine cases of multiple pregnancy with fetuses of opposite sex and fused chorionic vessels, giving forty-eight presumptive freemartins. There was one such multiple pregnancy with no chorionic fusion, and twelve multiple pregnancies where the fetuses were all of the same sex (Table 3). In five pregnancies, one or more fetuses had died at an early stage (Table 4).

\section{Assessment of the freemartin condition}

Macroscopic changes allowing one to diagnose the freemartin condition do not become very obvious before Day 60 (see Plate 2). Histological changes in the ovaries or the Müllerian ducts permit a diagnosis after Day 49.

Since the chorionic vascular connections between twin fetuses are already well established in 39-day-old pregnancies, it was assumed that in heterosexual pairs or groups of fetuses with clear vascular anastomoses, the females were presumptive freemartins.

In eleven groups of fetuses of mixed sexes, chromosomal chimaerism was observed in the liver (XY in females and XX in males). This was considered as proof that the predisposing cause of freemartinism was present, even if no anomalies were observed because the females were under 50 days old. However, no chimaerism was demonstrable in two presumptive freemartin fetuses aged

\section{EXPLANATION OF PLATES 2 TO 4}

\section{PLATE 2}

Dissections showing the gonads, the genital ducts, and the external genitalia of calf fetuses (enlargement $\times 3 \cdot 3$ for all fetuses).

Upper row: 52-day-old fetuses. On the left, normal male (fetus VT 33); notice that the testes are less elongated than the ovaries in the female and that the penis has already moved beyond the genital swellings. In the middle, normal female (fetus VT 31). On the right, freemartin $\mathrm{A}$ (fetus $934 \mathrm{~F} 2$ ) with stunted ovaries.

Middle row: 60 -day-old fetuses. On the left, normal male; notice the scrotum and the long ano-genital distance; in the middle, normal female. On the right, freemartin (F.M.) (fetus 375 F 1), whose ovaries show the average degree of inhibition (ovarian volume approximately on the regression line calculated in Text-fig. 6); it belonged to a triplet $\left(1 \delta^{*}+2\right.$ o ) pregnancy (Table 7$)$.

Lower row: Variations in 60- or 61-day-old fetuses. On the left side, normal 61-day-old female from a male-female twin pregnancy with no fusion (N.F.) of the chorions and no vascular connections (fetus $1134 \mathrm{~F} 1$ ). In the middle, 60 -day-old freemartin J (fetus 1225 F 3) showing slight ovarian inhibition; it belonged to a quadruplet $\left(1 \delta^{*}+3 \%\right)$ pregnancy. On the right side, 61-day-old freemartin L (fetus 572 F 3) showing a severe degree of ovarian inhibition; it belonged to a triplet $(1 \delta+2$ of pregnancy (Table 7$)$.

\section{PLATE 3}

Histological sections through presumptive ovaries.

On the left side, 45-day-old fetuses (enlargement $\times 80$ ). Top: Control female (fetus VT 17). In the middle: Presumptive freemartin (fetus S 247 F 4). Lower: Littermate presumptive freemartin (fetus S 247 F 3).

On the right side, 60-day-old fetuses (enlargement $\times 40$ ). Top: Control female (No. 890). So-called 'Pflüger's cords' are developing. In the middle: Freemartin J (1225 F 3), also illustrated in Plate 2, displaying a mild degree of ovarian inhibition. Lower: Freemartin G (375 F 2) whose gonads are severely inhibited (see Text-fig. 7).

\section{PLATE 4}

Histological sections through the upper Müllerian duct, on the margin of the mesonephros, and near the Wolffian duct, on Days 49 (upper horizontal row), 52 (middle horizontal row) and 60 (lower horizontal row). In each row, the male is on the left, the female in the middle and the freemartin on the right. (Freemartins $1118 \mathrm{~F} 1,1152 \mathrm{~F} 2$ $+Q$ and $375 \mathrm{~F} 1=\mathrm{F}$ ). On Day 49, the Müllerian ducts still are very similar in males, in females and in freemartins. Notice their parallel regression in freemartins and in males on Days 52 and 60. W $=$ Wolffian duct (enlargement $\times 310$ ). 
TABLE 3

NUMBER AND LENGTH OF MALE AND FEMALE FETUSES IN BOVINE MULTIPLE PREGNANCIES OF VARYING AGES

\begin{tabular}{|c|c|c|c|}
\hline $\begin{array}{c}\text { Gestational age } \\
\text { (days) }\end{array}$ & Animal no. & $\begin{array}{l}\text { No. and sex } \\
\text { of fetuses }\end{array}$ & $\begin{array}{l}\text { Length } \\
(\mathrm{mm})\end{array}$ \\
\hline $\begin{array}{l}\text { (1) Fetuses of } \\
\text { opposite sex } \\
39\end{array}$ & 1438 & $19+1 \delta^{(1)}$ & \\
\hline 44 & B 64 & $3 q+15$ & $25,26,27,25$ \\
\hline 45 & $\begin{array}{l}\text { S } 247 \\
1195 \\
\text { B } 73\end{array}$ & $\begin{array}{l}39+2 \sigma^{*} \\
10+2 \delta^{*(2)} \\
20+1 \delta^{* *(3)}\end{array}$ & $\begin{array}{l}28,32,29,31,31 \\
27,26,26 \\
28,28,28\end{array}$ \\
\hline 48 & $\begin{array}{l}\text { C } 10 \\
\text { S } 321\end{array}$ & $\begin{array}{l}2 q+20 \\
2 q+10\end{array}$ & $\begin{array}{l}30,30,30,29 \\
33,34,33\end{array}$ \\
\hline 49 & $\begin{array}{l}867 \\
1118 \\
1884 \\
874 \\
872\end{array}$ & $\begin{array}{l}19+3 \sigma^{*} \\
19+1 \sigma^{*} \\
29+1 \sigma^{*} \\
19+2 \sigma^{*} \\
19+2 \sigma^{*}\end{array}$ & $\begin{array}{l}34,32,33,33 \\
31,33 \\
32,32,31 \\
34,35,37\end{array}$ \\
\hline 52 & $\begin{array}{l}934 \\
1152 \\
745 \\
1364\end{array}$ & $\begin{array}{l}19+20^{*} \\
19+10^{*} \\
19+20^{*} \\
19+10^{*}\end{array}$ & $\begin{array}{l}44,47,46 \\
46,46 \\
39,43,41\end{array}$ \\
\hline 53 & VT 208 & $19+1 \delta^{(1)}$ & 52,52 \\
\hline 54 & 975 & $3 q+1 \sigma^{x}$ & $44,45,46$ \\
\hline 59 & 1335 & $19+10^{*}$ & \\
\hline 60 & $\begin{array}{l}\text { C } 37 \\
375 \\
\text { C } 43 \\
1225\end{array}$ & $\begin{array}{l}19+10^{*} \\
20+10^{*} \\
49+20^{*} \\
39+10^{*}\end{array}$ & $\begin{array}{l}70,65 \\
60,59,61 \\
60,61,62,64\end{array}$ \\
\hline 61 & $\begin{array}{l}572 \\
\text { C } 14 \\
1134\end{array}$ & $\begin{array}{l}29+1 \sigma^{*} \\
19+1 \sigma^{*} \\
19+1 \sigma^{(4)}\end{array}$ & $\begin{array}{l}70,72,71 \\
59,60 \\
67,69\end{array}$ \\
\hline 62 & $\begin{array}{l}\text { G } 63 \\
\text { G } 11 \\
\text { C } 88 \\
\text { C } 15\end{array}$ & $\begin{array}{l}2 q+1 \sigma^{* *(3)} \\
1 q+1 \delta^{*} \\
1 q+2 \delta^{*} \\
2 q+1 \delta^{*}\end{array}$ & $\begin{array}{l}71,68,70 \\
63,68 \\
75,80,65 \\
64,64,68\end{array}$ \\
\hline $\begin{array}{l}\text { (2) Fetuses all } \\
\text { male } \\
48\end{array}$ & 1058 & $4 \hat{0}$ & \\
\hline 49 & 1153 & $50^{*}$ & $33,31,29,30,29$ \\
\hline 56 & $\begin{array}{l}692 \\
731\end{array}$ & $\begin{array}{l}2 \sigma \\
2 \sigma\end{array}$ & 59,58 \\
\hline 57 & $\begin{array}{l}1024 \\
\text { L. } 17\end{array}$ & $\begin{array}{l}2 \delta \\
4 \delta\end{array}$ & $\begin{array}{l}53,55 \\
58,53,57,60\end{array}$ \\
\hline 58 & 768 & $2 \sigma^{*}$ & 62,65 \\
\hline $\begin{array}{l}\text { (3) Fetuses all } \\
\text { female } \\
50\end{array}$ & $\begin{array}{l}1185 \\
1033\end{array}$ & $\begin{array}{l}29 \\
49\end{array}$ & $\begin{array}{l}37,38 \\
34,34,34,34\end{array}$ \\
\hline 58 & 772 & 29 & 61,54 \\
\hline 60 & 591 & $3 q$ & $67,66,71$ \\
\hline 61 & C 1 & 29 & 73,77 \\
\hline
\end{tabular}

* XX/XY chimaerism verified. 
39 and 52 days; in the latter (No. 1364 F2, Table 7), 295 mitoses all showed an $\mathrm{XX}$ chromosome constitution although characteristic freemartin changes were obvious in the sections; the 39-day-old pair were too young to show any freemartin effect.

Finally, in one 61-day-old pair of opposite-sexed fetuses (No. 1134 F1, Tables 3 and 4, Plate 2), there was no chorionic fusion, no freemartin effect and no $\mathrm{XX} / \mathrm{XY}$ chimaerism.

\section{Gonadal volume}

The volume of the gonads was measured by the paper weight method from serial histological sections. Drawings of the ovarian contour were made by projection of sections at every $250 \mu \mathrm{m}$; the intervening volume was considered

TABLE 4

LENGTH OF SURVIVING AND DEAD FETUSES IN BOVINE MULTIPLE PREGNANGIES OF VARYING AGES

\begin{tabular}{|c|c|c|c|c|}
\hline $\begin{array}{c}\text { Gestational age } \\
\text { (days) }\end{array}$ & Animal no. & $\begin{array}{c}\text { No. of } \\
\text { surviving } \\
\text { fetuses (length in } \mathrm{mm})\end{array}$ & $\begin{array}{c}\text { No. of } \\
\text { dead fetuses } \\
\text { (length in } \mathrm{mm} \text { ) }\end{array}$ & $\begin{array}{r}X X \mid X Y \\
\text { chimaerism }\end{array}$ \\
\hline 48 & 943 & 1* & $10(5$ to 10$)$ & $\begin{array}{l}\mathrm{XY} \text { cells present in } \\
\text { female }\end{array}$ \\
\hline $\begin{array}{l}60 \\
61 \\
74 \\
80\end{array}$ & $\begin{array}{l}1378 \\
\text { C } 38 \\
\text { C } 53 \\
\text { C } 94\end{array}$ & $\begin{array}{l}1 \text { if }(60 \mathrm{~mm}) \\
2 \delta^{\prime}(60 \text { and } 65 \mathrm{~mm}) \\
10+(105 \mathrm{~mm}) \\
10(270 \mathrm{~mm})\end{array}$ & $\begin{array}{l}2(6 \text { to } 7) \\
4(7 \text { to } 15) \\
2(5 \text { and } 7) \\
10+19+1 ?(28,30,24)\end{array}$ & $\begin{array}{l}\text { No } X Y \text { cells present } \\
\text { Not studied } \\
\text { No } X Y \text { cells present } \\
\text { Not studied }\end{array}$ \\
\hline
\end{tabular}

* Gonads not studied histologically.

† Normal female.

as a cylinder with an area equal to the half sum of the areas of the two adjacent sections. Tables 5 to 7 give the volumes of one or both the gonads of normal fetuses and of freemartins. In Text-figs. 1, 2 and 3, the average volume of one gonad is plotted for those fetuses in which both gonads were available.

\section{Diameter of the upper Mïllerian ducts}

The Müllerian ducts are made of a cylinder of epithelial cells enclosed in the mesenchyme of the mesonephros and of the genital folds. Only the epithelium was investigated in this study, and diameters refer to the external diameter of the epithelial cylinder.

In normal male fetuses and in freemartins, the regression of the Müllerian ducts starts adjacent to the mesonephros. For this reason, the part was studied in detail. In females, it has a fairly constant diameter, excepting at the ostial end which is somewhat dilated. In males and in freemartins, the reduction in

Notes to Table 3:

(1) Spontaneous twin pregnancy.

(2) Vascular anastomosis was present between the female and one male; no anastomosis between this group and the other male.

(3) Three additional fetuses were dead ( $\leqslant 7 \mathrm{~mm}$, sex unknown).

(4) No vascular anastomosis; no freemartin effect; no $\mathrm{XX} / \mathrm{XY}$ chimaerism.

(5) No histological study was made of the genital tract of these freemartins. 
A. Jost et al.

TABLE 5

TESTIGULAR VOLUME AND DIAMETER OF MÜLLERIAN DUGTS IN BOVINE MALE FETUSES

\begin{tabular}{|c|c|c|c|c|c|c|}
\hline $\begin{array}{c}\text { Gestational age } \\
\text { (days) }\end{array}$ & Animal no. & $\begin{array}{l}\text { Length } \\
(m m)\end{array}$ & \multicolumn{2}{|c|}{$\begin{array}{l}\text { Testis volume } \\
\left(\mathrm{mm}^{3}\right)\end{array}$} & \multicolumn{2}{|c|}{ Müllerian duct diameter } \\
\hline $\begin{array}{l}\text { Pregnancies with } \\
\text { singletons } \\
\quad 42\end{array}$ & VT 5 & 28 & $0 \cdot 27$ & 0.39 & - & - \\
\hline 43 & $\begin{array}{l}\text { VT } 123 \\
1429 \\
\text { C } 16\end{array}$ & $\frac{21}{30}$ & $\begin{array}{l}0.24 \\
0.64 \\
0.49\end{array}$ & $\begin{array}{l}0.23 \\
0.56 \\
0.47\end{array}$ & $\bar{z}$ & $\bar{z}$ \\
\hline 45 & $\begin{array}{l}\text { VT } 19 \\
\text { B } 81\end{array}$ & $\begin{array}{l}35 \\
34\end{array}$ & $\begin{array}{l}1.10 \\
0.82\end{array}$ & $\begin{array}{l}0.97 \\
0.90\end{array}$ & $\begin{array}{l}64 \cdot 3 \\
62 \cdot 2\end{array}$ & $\begin{array}{l}62 \cdot 1 \\
64 \cdot 2\end{array}$ \\
\hline 46 & 1092 & - & - & - & $55 \cdot 3$ & $55 \cdot 3$ \\
\hline 47 & $\begin{array}{l}\text { B } 98 \\
\text { VT } 46\end{array}$ & $\begin{array}{l}33 \\
39\end{array}$ & $\stackrel{1.03}{-}$ & 0.84 & $\begin{array}{l}57.8 \\
61.9\end{array}$ & $\begin{array}{l}56 \cdot 1 \\
62 \cdot 2\end{array}$ \\
\hline 49 & $\begin{array}{l}\text { VT } 10 \\
\text { VT } 16 \\
\text { VT } 15\end{array}$ & $\begin{array}{l}40 \\
40 \\
43\end{array}$ & $\begin{array}{l}0.94 \\
1.35 \\
1.64\end{array}$ & $\begin{array}{l}0.90 \\
1.44 \\
1.45\end{array}$ & $\frac{55.5}{54.8}$ & $\frac{55.0}{55.4}$ \\
\hline 50 & VT 76 & 40 & - & - & 40.5 & $39 \cdot 0$ \\
\hline 52 & $\begin{array}{l}822 \\
1039 \\
\text { VT } 33 \\
\text { VT } 207\end{array}$ & $\begin{array}{l}39 \\
47 \\
48 \\
-\end{array}$ & $\begin{array}{l}1.61 \\
1.40 \\
1.31 \\
-\end{array}$ & $\begin{array}{l}1.55 \\
1.28 \\
1.33 \\
-\end{array}$ & $\begin{array}{l}32 \cdot 4 \\
31 \cdot 0 \\
33 \cdot 5 \\
29 \cdot 0\end{array}$ & $\begin{array}{l}32 \cdot 4 \\
31 \cdot 4 \\
33 \cdot 8 \\
31 \cdot 2\end{array}$ \\
\hline 53 & VT 223 & 47 & - & - & $23 \cdot 2$ & $25 \cdot 0$ \\
\hline 54 & $\begin{array}{l}\text { VT } 225 \\
\text { VT } 210\end{array}$ & 43 & $=$ & 二 & $\begin{array}{l}34 \cdot 8 \\
20 \cdot 3\end{array}$ & $\begin{array}{l}34.9 \\
20.0\end{array}$ \\
\hline & VT 219 & 40 & - & - & 33.0 & $33 \cdot 1$ \\
\hline 55 & $\begin{array}{l}\text { VT } 205 \\
\text { VT } 92\end{array}$ & $\begin{array}{l}50 \\
58\end{array}$ & 二 & $\bar{z}$ & $\begin{array}{l}31 \cdot 3 \\
25 \cdot 6\end{array}$ & $\begin{array}{l}30 \cdot 2 \\
25.8\end{array}$ \\
\hline 56 & $\begin{array}{l}\text { VT } 13 \\
\text { VT } 42\end{array}$ & $\begin{array}{l}64 \\
64\end{array}$ & $\begin{array}{l}2 \cdot 80 \\
3 \cdot 31\end{array}$ & $\begin{array}{l}2 \cdot 65 \\
3 \cdot 21\end{array}$ & $\begin{array}{l}25 \cdot 8 \\
28.9\end{array}$ & $\begin{array}{l}25 \cdot 4 \\
24 \cdot 3\end{array}$ \\
\hline 58 & $\begin{array}{l}\text { VT } 35 \\
\text { VT } 37\end{array}$ & $\begin{array}{l}70 \\
71\end{array}$ & $=$ & $=$ & $\begin{array}{l}25 \cdot 1 \\
20.0\end{array}$ & $\begin{array}{l}16 \cdot 8 \\
17.1\end{array}$ \\
\hline 59 & $C_{41}$ & 65 & - & - & $14 \cdot 5$ & $15 \cdot 7$ \\
\hline 60 & $\begin{array}{l}\text { VT } 24 \\
\text { VT 29 } \\
\text { VT 41 }\end{array}$ & $\begin{array}{l}74 \\
77 \\
80\end{array}$ & $\begin{array}{l}2 \cdot 61 \\
3 \cdot 13 \\
2 \cdot 59\end{array}$ & $\begin{array}{l}2.96 \\
2.88 \\
2.85\end{array}$ & $\begin{array}{l}21 \cdot 7 \\
20 \cdot 2 \\
-\end{array}$ & $\begin{array}{l}22.5 \\
18.5\end{array}$ \\
\hline 63 & VT 21 & 87 & - & - & 4.2 & $2 \cdot 1$ \\
\hline $\begin{array}{l}\text { Pregnancies with } \\
\text { multiple male } \\
\text { fetuses } \\
48\end{array}$ & & - & & - & 55.3 & \\
\hline 49 & $\begin{array}{r}1153 \text { F } 1 \\
\text { F } 2 \\
F \text { 3 } \\
\text { F } 5\end{array}$ & $\begin{array}{l}33 \\
31 \\
29 \\
29\end{array}$ & $\bar{z}$ & $\bar{z}$ & $\begin{array}{l}49 \cdot 8 \\
54 \cdot 3 \\
58 \cdot 4 \\
52 \cdot 2\end{array}$ & $\begin{array}{l}53 \cdot 1 \\
53 \cdot 5 \\
55.0\end{array}$ \\
\hline 56 & $731 \quad \begin{array}{l}F \\
F\end{array}$ & 二 & - & $=$ & $\begin{array}{l}26 \cdot 6 \\
25 \cdot 2\end{array}$ & $\begin{array}{l}27 \cdot 6 \\
25.9\end{array}$ \\
\hline 57 & 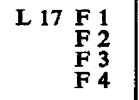 & $\begin{array}{l}58 \\
53 \\
57 \\
60\end{array}$ & $\bar{z}$ & $\bar{z}$ & $\begin{array}{l}13 \cdot 4 \\
26 \cdot 9 \\
28.9 \\
25 \cdot 4\end{array}$ & $\begin{array}{l}17 \cdot 2 \\
25.1 \\
27.5 \\
25.9\end{array}$ \\
\hline $\begin{array}{l}\text { Male co-twins to } \\
\text { freemartins } \\
45\end{array}$ & S 247F 1 & 31 & 0.81 & 0.83 & $55 \cdot 6$ & $61 \cdot 0$ \\
\hline & 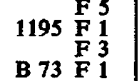 & $\begin{array}{l}31 \\
26 \\
26 \\
28\end{array}$ & $\begin{array}{l}0.71 \\
0.31 \\
0.46\end{array}$ & $\begin{array}{l}0.68 \\
0.43 \\
0.46\end{array}$ & $\begin{array}{l}\overline{62 \cdot 3} \\
64 \cdot 6 \\
51 \cdot 6\end{array}$ & $\begin{array}{l}6 \overline{67.0} \\
68.9 \\
54.7\end{array}$ \\
\hline 48 & $\begin{array}{l}C 10 \text { F } 2 \\
S_{321} F_{3}\end{array}$ & $\begin{array}{l}30 \\
29 \\
33\end{array}$ & $\overline{0.86}$ & $\frac{\overline{0}}{0.90}$ & $\overline{\overline{65 \cdot 6}}$ & $\begin{array}{l}59.5 \\
57.4 \\
60.1\end{array}$ \\
\hline 49 & 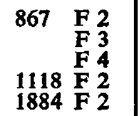 & $\begin{array}{l}32 \\
33 \\
33 \\
33 \\
31\end{array}$ & $\begin{array}{l}0.90 \\
= \\
=\end{array}$ & $\begin{array}{l}0.80 \\
= \\
=\end{array}$ & $\begin{array}{l}63 \cdot 7 \\
59 \cdot 3 \\
57 \cdot 8 \\
59 \cdot 5\end{array}$ & $\begin{array}{l}64 \cdot 8 \\
60.1 \\
63.9 \\
59 \cdot 5 \\
57.7\end{array}$ \\
\hline 52 & $\begin{array}{ll}934 & \text { F 1 } \\
745 & \text { F 3 } \\
& \text { F 1 } \\
& \text { F 2 }\end{array}$ & $\begin{array}{l}47 \\
46 \\
43 \\
41\end{array}$ & $\begin{array}{l}2 \cdot 23 \\
2 \cdot 17 \\
=\end{array}$ & $\begin{array}{l}2.08 \\
1.94 \\
=\end{array}$ & $\begin{array}{l}\overline{35.6} \\
30.8 \\
36.0\end{array}$ & $\begin{array}{l}36 \cdot 1 \\
30 \cdot 2 \\
-\end{array}$ \\
\hline 53 & VT 208 F 1 & 52 & - & - & 30.0 & $30 \cdot 0$ \\
\hline 54 & 975 F 2 & 46 & 2.07 & $2 \cdot 21$ & $34 \cdot 2$ & $36 \cdot 1$ \\
\hline 60 & 1225 F 4 & 64 & - & - & $27 \cdot 4$ & $26 \cdot 4$ \\
\hline 62 & C 11 F 1 & 68 & 3.07 & 2.98 & 18.0 & $18 \cdot 0$ \\
\hline
\end{tabular}


TABLE 6

OVARIAN VOLUME AND DIAMETER OF UPPER MÜLLERIAN DUGTS IN BOVINE FEMALE FETUSES

\begin{tabular}{|c|c|c|c|c|c|c|}
\hline \multirow{2}{*}{$\begin{array}{l}\begin{array}{l}\text { Gestational age } \\
\text { (days) }\end{array} \\
\begin{array}{l}\text { Pregnancies } \\
\text { with singletons } \\
42\end{array}\end{array}$} & Animal no. & $\begin{array}{c}\text { Length } \\
(m m)\end{array}$ & \multicolumn{2}{|c|}{$\begin{array}{l}\text { Ovarian volume } \\
\left(\mathrm{mm}^{3}\right) \\
\text { Right } \quad \text { Left }\end{array}$} & \multicolumn{2}{|c|}{$\begin{array}{l}\text { Müllerian duct diameter } \\
\text { Right }{ }^{(\mu m)} \text { Left }\end{array}$} \\
\hline & $\begin{array}{l}\text { VT } 9 \\
\text { VT } 8 \\
\text { VT } 129\end{array}$ & $\overline{-}$ & $\begin{array}{l}0.29 \\
0.23 \\
0.17\end{array}$ & $\begin{array}{l}0.33 \\
0.25 \\
0.18\end{array}$ & 二 & E \\
\hline 43 & C 23 & 23 & 0.21 & 0.20 & $一$ & - \\
\hline 45 & $\begin{array}{l}\text { VT } 17 \\
\text { B } 60\end{array}$ & $\begin{array}{l}32 \\
31\end{array}$ & $\begin{array}{l}0.49 \\
0.55\end{array}$ & $\begin{array}{l}0.51 \\
0.49\end{array}$ & $\begin{array}{l}54 \cdot 1 \\
66 \cdot 3\end{array}$ & $\begin{array}{l}54 \cdot 1 \\
63 \cdot 8\end{array}$ \\
\hline 49 & $\begin{array}{l}\text { VT } 20 \\
876 \\
811\end{array}$ & $\frac{40}{33}$ & $\begin{array}{l}0.98 \\
0 \cdot 79 \\
-\end{array}$ & $\begin{array}{c}0.85 \\
0.72 \\
-\end{array}$ & $\begin{array}{l}55 \cdot 1 \\
60 \cdot 5 \\
62 \cdot 5\end{array}$ & $\begin{array}{l}56 \cdot 6 \\
59 \cdot 3 \\
61 \cdot 4\end{array}$ \\
\hline 51 & VT 201 & 38 & - & - & $52 \cdot 6$ & $52 \cdot 6$ \\
\hline 52 & $\begin{array}{l}895 \\
753 \\
\text { VT } 31\end{array}$ & $\begin{array}{l}37 \\
37 \\
44\end{array}$ & $\begin{array}{l}1 \cdot 12 \\
1.55 \\
1 \cdot 09\end{array}$ & $\begin{array}{l}1.12 \\
1.50 \\
1.08\end{array}$ & $\begin{array}{l}44 \cdot 5 \\
46 \cdot 9 \\
47 \cdot 5\end{array}$ & $\begin{array}{l}46 \cdot 9 \\
47 \cdot 5 \\
47 \cdot 3\end{array}$ \\
\hline 53 & $\begin{array}{l}\text { VT } 214 \\
\text { VT } 217\end{array}$ & $\begin{array}{l}44 \\
52\end{array}$ & 二 & - & $\begin{array}{l}45 \cdot 2 \\
44 \cdot 5\end{array}$ & $\begin{array}{l}44 \cdot 1 \\
44 \cdot 5\end{array}$ \\
\hline 55 & 906 & 50 & 1.39 & 1.42 & $42 \cdot 6$ & $43 \cdot 5$ \\
\hline 56 & $\begin{array}{l}\text { VT } 27 \\
\text { VT } 14 \\
765 \\
782 \\
853\end{array}$ & $\begin{array}{l}57 \\
61 \\
56 \\
53 \\
56\end{array}$ & $\begin{array}{l}2.24 \\
1.65 \\
1.79 \\
1.81 \\
-\end{array}$ & $\begin{array}{l}2.00 \\
1.51 \\
1.88 \\
1.75 \\
\end{array}$ & $\begin{array}{l}42 \cdot 8 \\
39 \cdot 5 \\
41 \cdot 3 \\
43 \cdot 2 \\
41 \cdot 5\end{array}$ & $\begin{array}{l}40 \cdot 6 \\
44.0 \\
41.9 \\
44.5 \\
45 \cdot 2\end{array}$ \\
\hline 58 & VT 36 & 71 & - & - & $49 \cdot 2$ & $41 \cdot 7$ \\
\hline 59 & VT 206 & 62 & $2 \cdot 55$ & $2 \cdot 14$ & $49 \cdot 6$ & $49 \cdot 7$ \\
\hline 60 & $\begin{array}{l}1373 \\
890 \\
\text { C } 11 \text { bis } \\
\text { VT } 98 \\
1378 \text { F }^{*}\end{array}$ & $\begin{array}{l}65 \\
59 \\
70 \\
60\end{array}$ & $\begin{array}{l}3 \cdot 14 \\
1.92 \\
2 \cdot 28 \\
1.96\end{array}$ & $\begin{array}{l}2.55 \\
1.91 \\
2 \cdot 16 \\
\overrightarrow{1.92}\end{array}$ & $\begin{array}{l}52 \cdot 7 \\
51.8 \\
50.4 \\
51.2 \\
51.8\end{array}$ & $\begin{array}{l}54 \cdot 2 \\
49 \cdot 0 \\
47 \cdot 4 \\
50 \cdot 8 \\
49 \cdot 0\end{array}$ \\
\hline 64 & VT 18 & 75 & - & - & $58 \cdot 6$ & $55 \cdot 6$ \\
\hline $\begin{array}{l}\text { Pregnancies } \\
\text { with multiple } \\
\text { female fetuses } \\
\text { with } \\
\text { anastomoses } \\
\quad 50\end{array}$ & $\begin{array}{r}1185 \text { F } 1 \\
\text { F } 2 \\
1033 \text { F } 1 \\
\text { F } 2 \\
\text { F } 3 \\
\text { F } 4\end{array}$ & $\begin{array}{l}37 \\
38 \\
34 \\
34 \\
34 \\
34\end{array}$ & $\begin{array}{l}= \\
= \\
=\end{array}$ & $\begin{array}{l}= \\
= \\
=\end{array}$ & $\begin{array}{l}47 \cdot 8 \\
49 \cdot 4 \\
50 \cdot 2 \\
45 \cdot 1 \\
54 \cdot 7 \\
49 \cdot 8\end{array}$ & $\begin{array}{l}45 \cdot 1 \\
5 \overrightarrow{53 \cdot 9} \\
45 \cdot 5 \\
52 \cdot 0 \\
46 \cdot 3\end{array}$ \\
\hline 60 & 591 F 3 & 71 & 3.28 & $3 \cdot 30$ & $53 \cdot 2$ & 50.7 \\
\hline 61 & $\begin{array}{ll}\text { C } 1 & F \\
& F 2\end{array}$ & $\begin{array}{l}73 \\
77\end{array}$ & $\begin{array}{l}3 \cdot 29 \\
3 \cdot 22\end{array}$ & $\begin{array}{l}3.28 \\
2.92\end{array}$ & $\begin{array}{l}58.0 \\
54 \cdot 4\end{array}$ & $\begin{array}{l}58 \cdot 8 \\
52 \cdot 0\end{array}$ \\
\hline $\begin{array}{l}\text { Pregnancies } \\
\text { with male- } \\
\text { female twins } \\
\text { without } \\
\text { anastomoses } \\
61\end{array}$ & 1134 F 1 & 67 & $2 \cdot 50$ & $2 \cdot 66$ & $51 \cdot 4$ & 53.6 \\
\hline
\end{tabular}

* Associated with small dead fetuses; no XX/XY chimaerism (see Table 4). 
TABLE 7

OVARIAN VOLUME AND DIAMETER OF UPPER MÜLLERIAN DUCTS IN BOVINE FREEMARTINS

\begin{tabular}{|c|c|c|c|c|c|c|}
\hline $\begin{array}{c}\text { Gestational age } \\
\text { (days) }\end{array}$ & Identification & $\begin{array}{l}\text { Length } \\
(\mathrm{mm})\end{array}$ & \multicolumn{2}{|c|}{$\begin{array}{l}\text { Ovarian volume } \\
\quad\left(\mathrm{mm}^{3}\right) \\
\text { Right }{ }_{\text {Left }}\end{array}$} & \multicolumn{2}{|c|}{$\begin{array}{c}\text { Müllerian diameter } \\
(\mu \mathrm{m})\end{array}$} \\
\hline 39 & 1438 F 1 & - & 0.12 & 0.12 & - & - \\
\hline 44 & $\begin{array}{r}\text { B } 64 \text { F } 2 \\
F 3 \\
\text { F } 4\end{array}$ & $\begin{array}{l}25 \\
26 \\
27\end{array}$ & $\begin{array}{l}0 \cdot 30 \\
0 \cdot 34 \\
0.24\end{array}$ & $\begin{array}{l}0.31 \\
0.32 \\
0.23\end{array}$ & E & - \\
\hline 45 & $\begin{array}{r}\text { S247 F } 2 \\
\text { F 3 } \\
\text { F 4 } \\
1195 \text { F } 2 \\
\text { B 73 F 3 } \\
\text { F } 4\end{array}$ & $\begin{array}{l}28 \\
32 \\
29 \\
27 \\
28 \\
28\end{array}$ & $\begin{array}{l}0.40 \\
0.50 \\
0.40 \\
0.37 \\
0.20\end{array}$ & $\begin{array}{l}0.37 \\
0 \cdot 47 \\
0 \cdot 38 \\
0 \cdot 30 \\
0.36 \\
0 \cdot 19\end{array}$ & $\begin{array}{l}58 \cdot 0 \\
57 \cdot 2 \\
56 \cdot 2 \\
62 \cdot 9 \\
53 \cdot 6 \\
63 \cdot 5\end{array}$ & $\begin{array}{l}53 \cdot 0 \\
62 \cdot 9 \\
55 \cdot 3 \\
63 \cdot 8 \\
54 \cdot 4 \\
61 \cdot 6\end{array}$ \\
\hline 48 & $\begin{array}{r}\text { G } 10 \text { F } 1 \\
\text { F } 4 \\
\text { S321 F } 1 \\
\text { F } 2\end{array}$ & $\begin{array}{l}30 \\
30 \\
33 \\
34\end{array}$ & $\frac{-}{\overline{0} 89}$ & $\begin{array}{l}0.48 \\
0.43 \\
0.80 \\
0.63\end{array}$ & $\begin{array}{l}\overline{-} \\
\overline{60 \cdot 8} \\
48 \cdot 6\end{array}$ & $\begin{array}{l}61 \cdot 8 \\
57 \cdot 4 \\
53 \cdot 0 \\
55 \cdot 9\end{array}$ \\
\hline 49 & $\begin{array}{lll}867 & \text { F } 1 \\
1118 & \text { F } 1 \\
1884 & \text { F 1 } \\
& \text { F3 } \\
874 & \text { F 1 } \\
872 & \text { F 2 }\end{array}$ & $\begin{array}{l}34 \\
31 \\
32 \\
32 \\
34 \\
-\end{array}$ & $\begin{array}{l}0.63 \\
- \\
\overline{-} \\
0.84 \\
0.82\end{array}$ & $\begin{array}{l}0.60 \\
0.69 \\
0.51 \\
0.51 \\
0.80 \\
0.69\end{array}$ & $\begin{array}{l}45 \cdot 3 \\
55 \cdot 9 \\
- \\
60 \cdot 1 \\
41 \cdot 7 \\
54 \cdot 0\end{array}$ & $\begin{array}{l}58 \cdot 1 \\
65 \cdot 8 \\
56 \cdot 2 \\
57 \cdot 4 \\
45 \cdot 2 \\
54 \cdot 4\end{array}$ \\
\hline 52 & $\begin{array}{l}934 \text { F } 2=A^{*} \\
1152 \text { F } 2=Q \\
745 \text { F } 3=R \\
1364 \text { F } 2=S\end{array}$ & $\begin{array}{l}44 \\
46 \\
39 \\
-\end{array}$ & $\begin{array}{l}0.63 \\
= \\
-\end{array}$ & $\begin{array}{l}0.52 \\
0.60 \\
0.36 \\
0.40\end{array}$ & $\begin{array}{l}34 \cdot 6 \\
32 \cdot 8 \\
33 \cdot 5 \\
37 \cdot 9\end{array}$ & $\begin{array}{l}32 \cdot 9 \\
32 \cdot 6 \\
36 \cdot 0 \\
39 \cdot 1\end{array}$ \\
\hline 53 & $\mathrm{VT} 208 \mathrm{~F} 2=\mathrm{T}$ & 52 & - & 0.46 & $36 \cdot 2$ & $34 \cdot 1$ \\
\hline 54 & $\begin{array}{r}975 \text { F } 1=B \\
\text { F } 3=C \\
\text { F } 4=D\end{array}$ & $\begin{array}{l}44 \\
45 \\
43\end{array}$ & $\begin{array}{l}0.97 \\
0.68 \\
0.77\end{array}$ & $\begin{array}{l}0.94 \\
0.72 \\
0.74\end{array}$ & $\begin{array}{l}41 \cdot 5 \\
34 \cdot 0 \\
34 \cdot 7\end{array}$ & $\begin{array}{l}39 \cdot 6 \\
32 \cdot 8 \\
34 \cdot 3\end{array}$ \\
\hline 59 & 1335 F $2=E$ & - & 0.68 & 0.66 & $29 \cdot 0$ & $27 \cdot 9$ \\
\hline 60 & $\begin{aligned} \text { C37 F } 2=U \\
375 \text { F } 1=F \\
\text { F } 2=G \\
\text { C 43 } 1=\mathrm{V} \\
\text { F } 2=W \\
\text { F } 3=\mathbf{X} \\
\text { F } 5=\mathbf{Y} \\
1225 \text { F } 1=\mathbf{H} \\
\text { F } 2=\mathbf{I} \\
\text { F } 3=\mathrm{J}\end{aligned}$ & $\begin{array}{l}70 \\
60 \\
59 \\
- \\
- \\
- \\
\overline{60} \\
61 \\
62\end{array}$ & $\begin{array}{l}\overline{-} \\
0.83 \\
0.66 \\
= \\
= \\
\overline{=} \\
1 \cdot 28 \\
1.10\end{array}$ & $\begin{array}{l}0.46 \\
0 \cdot 63 \\
0.46 \\
0.56 \\
0.54 \\
0 \cdot 61 \\
0.46 \\
1.09 \\
0.83 \\
1.32\end{array}$ & $\begin{array}{l}\overline{27 \cdot 0} \\
22 \cdot 6 \\
\overline{3} \\
\frac{-}{-} \\
34 \cdot 9 \\
31 \cdot 9 \\
30 \cdot 8\end{array}$ & $\begin{array}{l}22 \cdot 4 \\
24 \cdot 1 \\
21 \cdot 1 \\
26 \cdot 1 \\
32.5 \\
27.9 \\
23.9 \\
32.9 \\
30 \cdot 5 \\
28.7\end{array}$ \\
\hline 61 & $\begin{aligned} 572 \text { F } 2 & =K \\
\text { F } 3 & =\mathrm{L} \\
\text { G } 14 \mathrm{~F} 1 & =\mathrm{M}\end{aligned}$ & $\begin{array}{l}70 \\
72 \\
59\end{array}$ & $\begin{array}{l}1 \cdot 12 \\
0.40 \\
0.69\end{array}$ & $\begin{array}{l}1.02 \\
0.31 \\
0.59\end{array}$ & $\begin{array}{l}33 \cdot 3 \\
22 \cdot 3 \\
24 \cdot 2\end{array}$ & $\begin{array}{l}34 \cdot 0 \\
25 \cdot 7 \\
17 \cdot 6\end{array}$ \\
\hline 62 & $\begin{array}{r}\text { C } 11 \text { F } 2=N \\
\text { C } 15 \text { F } 2=O \\
\text { F } 3=P \\
\text { C } 88 \text { F } 1=Z\end{array}$ & $\begin{array}{l}63 \\
64 \\
64 \\
75\end{array}$ & $\begin{array}{l}0.87 \\
0.58 \\
0.42 \\
-\end{array}$ & $\begin{array}{l}0.69 \\
0.55 \\
0.38 \\
0.41\end{array}$ & $\begin{array}{r}22 \cdot 3 \\
10 \cdot 8 \\
2 \cdot 0 \\
-\end{array}$ & $\begin{array}{r}21 \cdot 0 \\
11 \cdot 4 \\
4 \cdot 4 \\
12 \cdot 3\end{array}$ \\
\hline
\end{tabular}

* The letters A to P refer to the cases shown in Text-fig. 7 and in Table 8. 
diameter after Day 49 is more pronounced in some parts than in others. In order to obtain an average value for these ducts, their diameter was measured at distances of $250 \mu \mathrm{m}$ in those sections of the mesonephros in which the gonads were also present (approximately seven measurements/side on Day 45

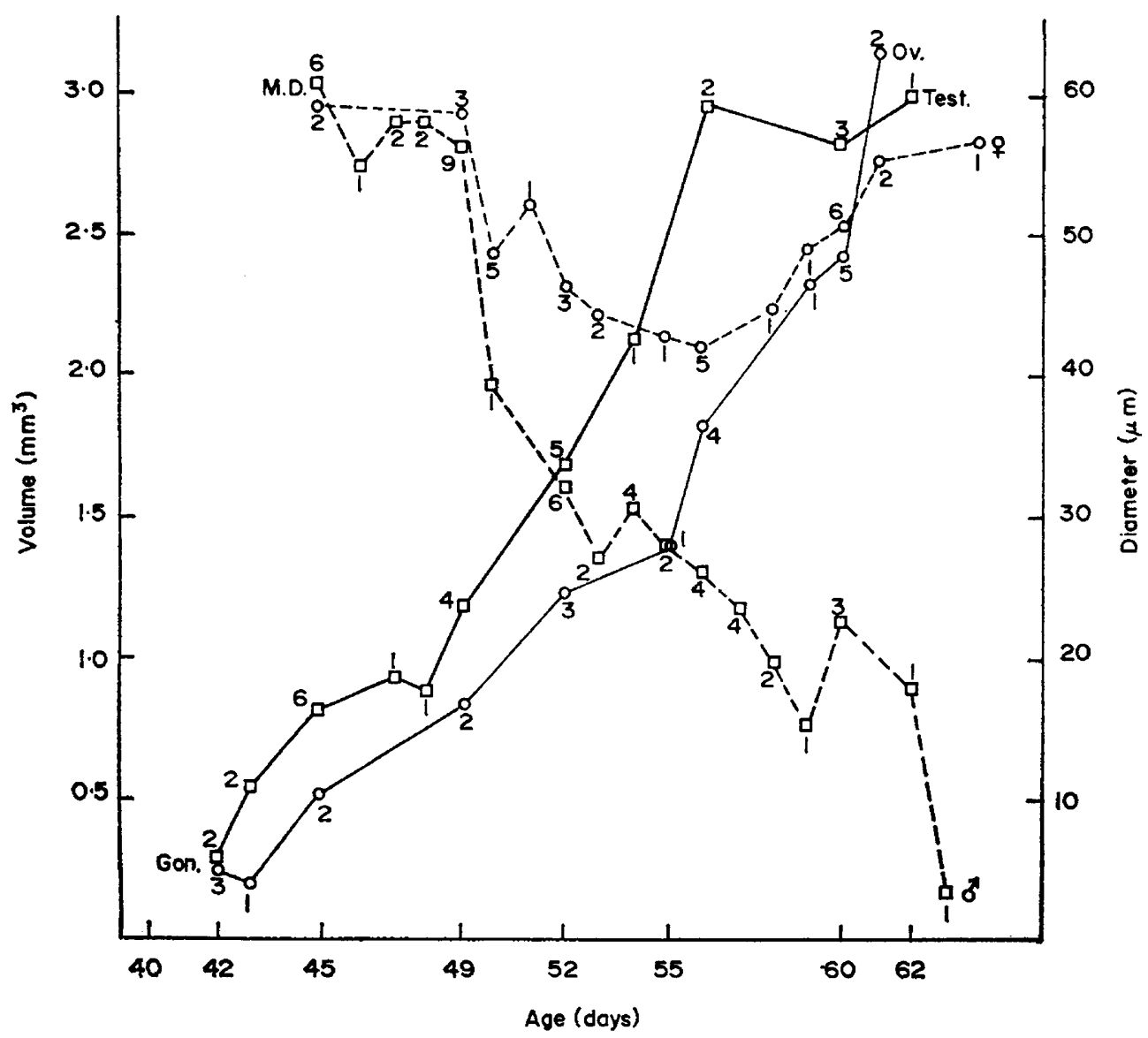

Text-Fig. 1. Variations in the volume $\left(\mathrm{mm}^{3}\right)$ of testes and ovaries (solid lines) and in the diameter $(\mu \mathrm{m})$ of the upper Müllerian ducts (dashed lines) of normal male and female calf fetuses between Days 42 and 63 ( $\square$, males; $O$, fermales). Each point gives the average value for the number of fetuses indicated near the point.

and twelve on Day 62). The mean value for the diameter of the Müllerian ducts was calculated and plotted in Text-figs. 1, 4 and 5 .

\section{Statistical calculations}

Correlation coefficients were calculated according to the Bravais-Pearson formula (see Simpson, Roe \& Lewontin, 1960). The significance of these correlation coefficients for $\mathrm{N}-2$ degrees of freedom was tested according to Fischer's tables. 


\section{RESULTS}

Gonadal volume

The average volume of both gonads was measured in twenty-nine freemartins between the ages of 39 and 62 days (Text-figs. 2 and 3); in seventeen other freemartins, only the volume of the left gonad was measured (Table 7). These volumes were compared with the gonadal volumes of normal fetuses

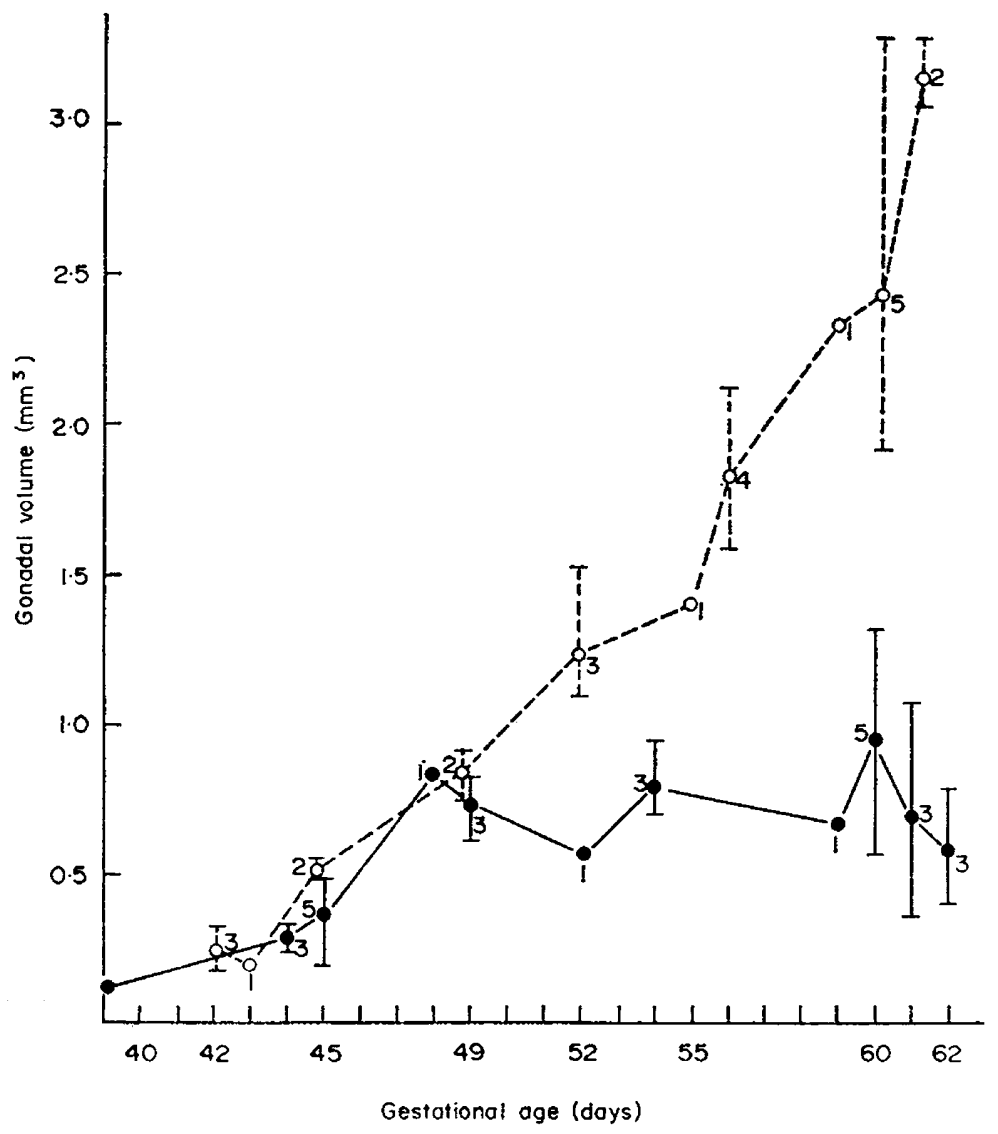

Texr-FIG. 2. Variations in gonadal volume according to gestational age in twenty-nine freemartins $(\bullet)$ as compared with normal females $(O)$. Each point on the curves gives the mean of the volumes of both gonads in all fetuses of the same age group. The numbers indicate the number of fetuses for each age and the vertical lines give the range of individual values.

from single pregnancies or from multiple pregnancies of one sex only or from the male members of opposite sexed pairs (Tables 5 and 6 ; Text-fig. 3 ).

Normal gonads. During the 39- to 62-day period there is approximately a tenfold increase in body weight and a threefold increase in crown-rump length. The increase in gonadal volume roughly parallels the body weight. In both sexes, the gonadal volume increases steadily with increasing age or length. Up to Day 56, the volume of the testes slightly exceeds that of the 
presumptive ovaries when plotted against fetal age (Text-fig. 1); this difference does not appear clearly when plotted against fetal length, since several females were slightly smaller than the males. In a few females of multiple pregnancies of one sex, the gonadal volume was normal relative to body size (Text-fig. 3). In both sexes, from Day 48 onwards, there was an indication of a lateral

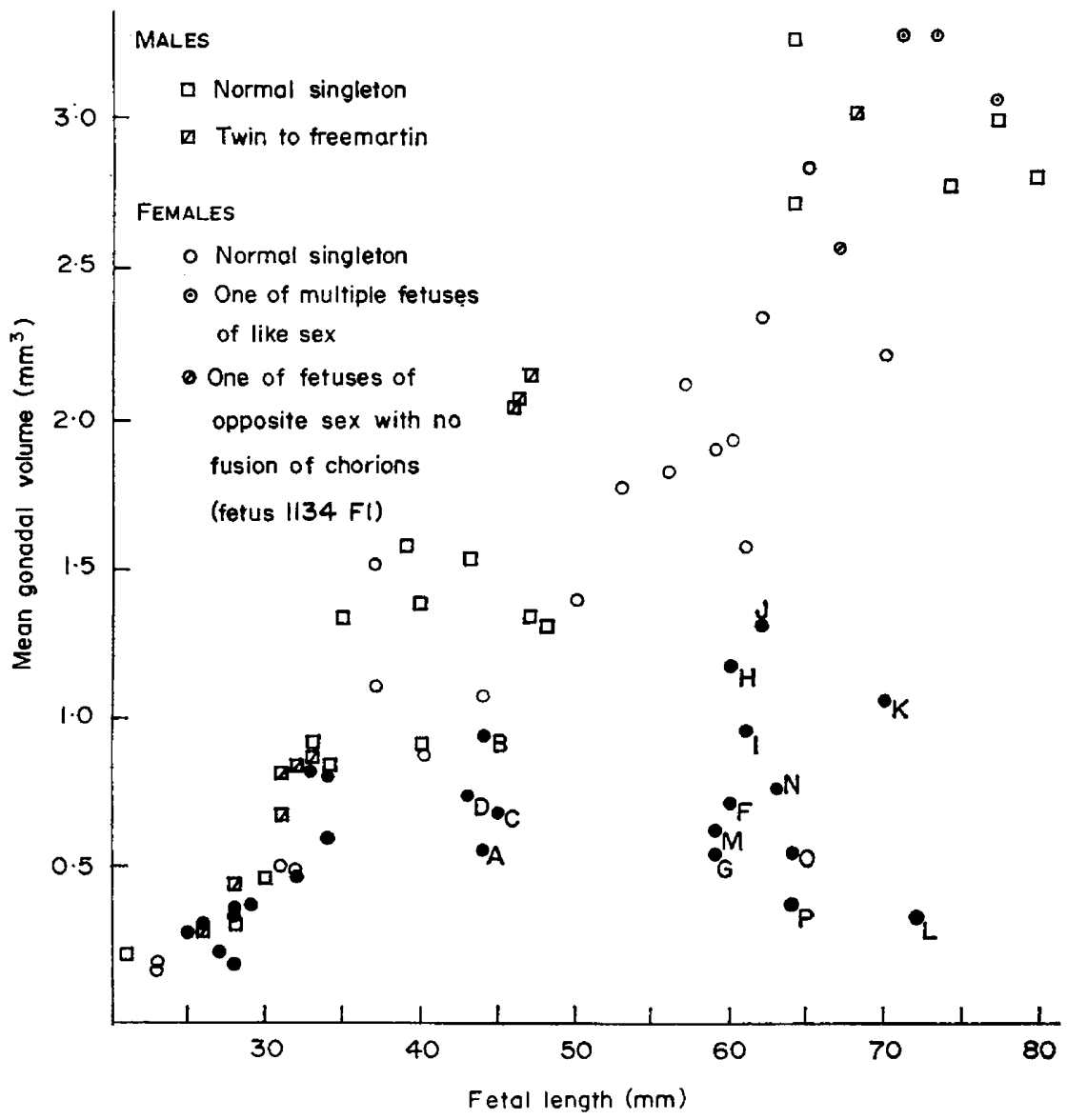

TeXT-FIG. 3. Correlation between mean gonadal volume $\left(\mathrm{mm}^{3}\right)$ and fetal length (mm). Freemartins: $\bullet$; the block letters are those used in Text-figs. 5 and 6 , and in Tables 7 and 8.

asymmetry, the left gonad frequently being somewhat smaller than the right, but this difference was inconsistent and is of very limited quantitative importance; it was observed in fourteen out of eighteen females and in eleven out of seventeen males (Tables 5 and 6 ).

Freemartin gonads. In freemartins, the gonadal volume increased in a fairly normal manner up to Day 48 or 49 ; thereafter, it practically stopped although there were some individual variations (see Text-fig. 2 and Plates 2 and 3 ). The same finding was apparent when gonadal volume was plotted against fetal length, since some of the early freemartins were rather small; the gonadal 
volume increased fairly normally up to a fetal size of $34 \mathrm{~mm}$ and, thereafter, it practically stopped (Text-fig. 3).

A similar picture was observed in the seventeen freemartins in which only the left gonad could be studied.

Lateral asymmetry between both gonads seems to be somewhat more frequent and pronounced in freemartins than in females or males: the left gonad was smaller than the right one in eighteen out of twenty freemartins collected between 48 and 62 days of age. The difference between the right and the left gonad was calculated for each fetus; the mean of these differences $\left(0.09 \mathrm{~mm}^{3}\right)$ differs significantly from zero $(P<0.001)$. This lateral asymmetry is relatively minor when compared with the differences from one freemartin to another (Table 7 and Plate 2).

In contrast to the small gonadal volume in freemartins, a normal ovarian volume was found in the 61-day-old female of the heterosexual pair with no fusion of the chorions (Fetus 1134 F1, Table 6 and Plate 2).

Male co-tzwins to freemartins. As shown in Table 5 and in Text-fig. 3, no difference in testicular volume appears between males from single pregnancies and males which were co-twins to freemartins, even when they were united to two or three freemartins.

\section{Diameter of the Müllerian ducts at the gonadal levels}

The diameter of the Müllerian ducts at the gonadal level was determined for each fetus (Tables 5 to 7 ) and the average values were plotted against age in Text-figs. 1 and 4.

Control fetuses. It should be noted that lateral asymmetry in the diameter of the Müllerian ducts is slight; no constant difference could be found between the right and the left Müllerian ducts in normal male or female fetuses (Tables 5 to 7 ). The mean values plotted in Text-figs. 1 and 4 are, therefore, representative. Between Days 45 and 49 (Text-figs. 1 and 4 and Plate 4), or before the fetus reaches a length of $35 \mathrm{~mm}$, there was no difference in the diameter of the Müllerian ducts in males and in females. In females, some transitory reduction in the upper Müllerian diameter occurs between Days 50 and 60. It never exceeds $30 \%$. In males, the diameter of the Müllerian ducts is conspicuously reduced on Day 52 and the reduction steadily continues until complete disappearance of the ducts. After Day 58, they become interrupted, which means that they are already absent in some sections and still present in other sections.

Freemartin fetuses. The diameter of the upper part of the Müllerian duct in the freemartin is of the same order of magnitude as in control females or males until Day 49 (Text-figs. 1 and 4) or until the stage of $35 \mathrm{~mm}$. A conspicuous drop is obvious on Day 52 and the reduction in diameter continues thereafter (Plate 4). From the stage of 52 days onward, the diameter of the upper Müllerian ducts in the freemartin is always smaller than in normal females.

There is an evident parallelism between the curves for males and for freemartins (Text-fig. 4), though the reduction of the Müllerian ducts in the freemartin is often somewhat less pronounced than in males of the same age. Overlapping of individual values is frequent. The significant drop in diameter occurs at a similar stage in males and in freemartins. 
In normal males, interruption of the Müllerian ducts at the mesonephric gonadal level has begun in eight out of nine fetuses over 58 days of age (the ducts were still continuous in one 60-day-old male). In freemartins, a similar interruption was present in only two out of ten of the 60-day-old group and in all the seven 61- and 62-day-old individuals. Since the reduction of the diameter probably started simultaneously in males and in freemartins, this would

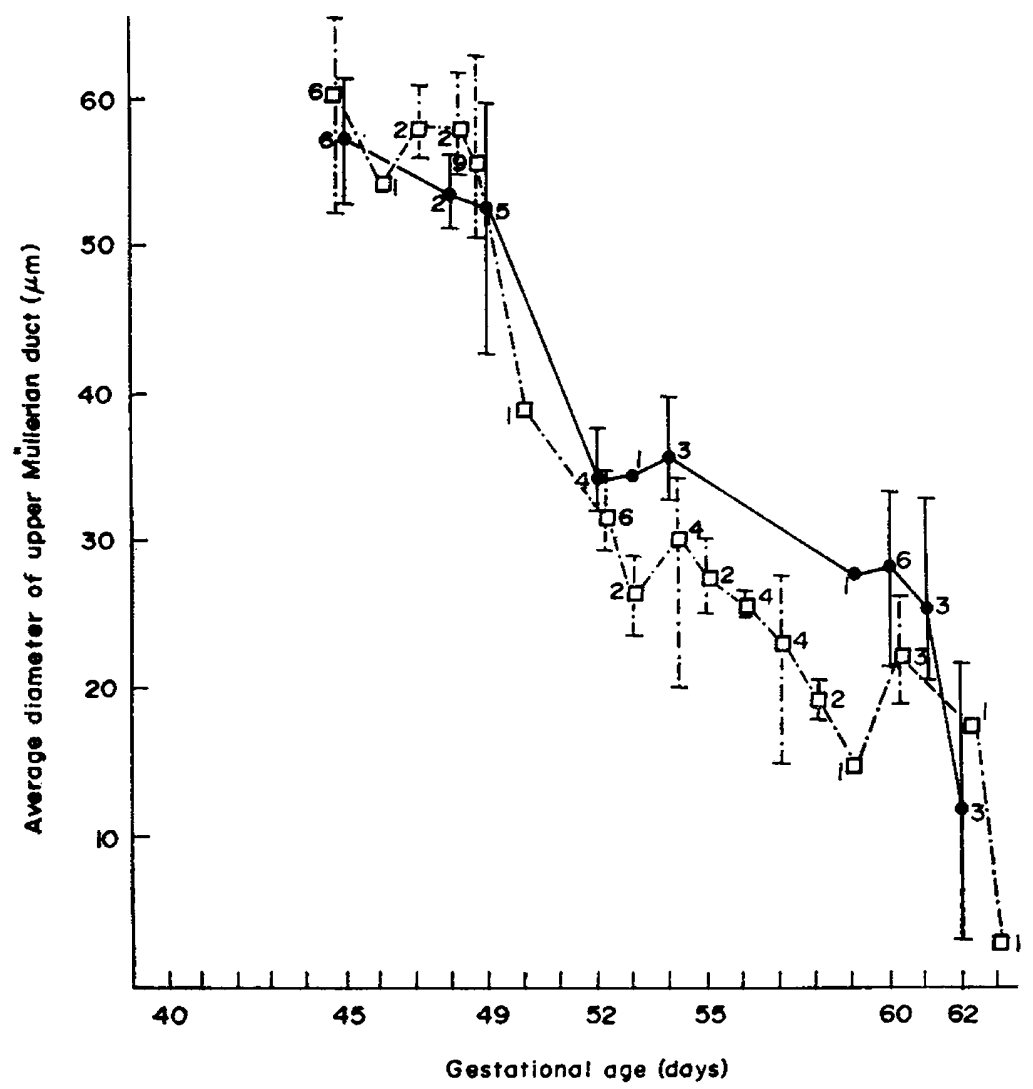

TExT-FIG. 4. Variations in average diameter of the upper Müllerian duct with gestational age in males (ㅁ) and in freemartins ( $\bullet$ ). Numbers indicate the number of fetuses for each age. The vertical lines indicate the range of individual values.

indicate a slower process of regression in the freemartins, producing a delay of 48 to $72 \mathrm{hr}$ in the interruption of the anterior part of their Müllerian ducts as compared with normal males.

As mentioned above, in control females no constant difference in the diameter of the right and left Müllerian ducts occurs; in freemartins, the left duct was the smaller in fourteen cases and the larger only in seven cases. There seems to be a trend for a slight lateral asymmetry in freemartins, but differences between animals are much greater (Table 7); the mean of the differences between the diameters of the right and of the left Müllerian duct is not significantly different from zero. 
In male co-twins to freemartins, the diameter of the Müllerian ducts was within the range for normal males. There was no delay in Müllerian retrogression in seven male co-twins to freemartins which were studied between the ages of 52 to 62 days.

\section{Correlation between ovarian and Müllerian inhibition}

From Text-figs. 2 and 4 , it can be seen that there is a chronological link between ovarian and Müllerian inhibition: both become obvious between Days 48 and 52.

An attempt was made to verify a possible correlation between the degrees of ovarian and of Müllerian inhibition.

(1) In the group of twenty 48- to 62-day-old freemartins in which both the right and the left gonadal volume was measured, the average gonadal volume in each fetus can be considered independent of the age of the fetus. The correlation coefficient between ovarian volume $(Y)$ and age $(x)$ was $r=+0.02$ which does not significantly differ from 0 , and the equation of the regression line was $\mathrm{Y}=0.703+0.001 \mathrm{x}$, where $\mathrm{Y}$ is expressed in $\mathrm{mm}^{3}$ and $\mathrm{x}$ in days (Text-fig. 5).

A similar result was obtained when the volume of the left ovary only was considered in thirty-six freemartins aged 48 to 62 days. The correlation coefficient between left ovarian volume $\mathrm{Y}$ and the age $(\mathrm{x})$ was not significantly different from $0(\mathrm{r}=+0.08)$.

(2) In the group of twenty-one 52- to 62-day-old freemartins in which both the right and the left Müllerian ducts were studied, the average diameter of the upper Müllerian ducts can be considered to decrease linearly. There is a negative correlation $(\mathrm{r}=-0.69, P<0.001)$ between diameter of the Müllerian ducts $(y)$ and age of the fetus $(x)$. A straight regression line could be calculated $(\mathrm{y}=118.656-1.562 \mathrm{x}$, where $\mathrm{y}$ is expressed in $\mu \mathrm{m})$.

The same result was observed when the diameter of the left Müllerian duct was studied in twenty-six freemartins aged 52 to 62 days $(\mathrm{r}=-0.68, P<0.001)$.

(3) Individual values for ovarian volume and for Müllerian diameter were then compared with the calculated theoretical values on the regression lines for the sixteen freemartins (A to P) studied between Days 52 to 62 where the ovaries and Müllerian ducts were studied on both sides (Text-fig. 5). A point above the regression line corresponds to a degree of inhibition lower than average, and vice versa. Text-figure 5 shows that in general there is a correlation between the degree of inhibition of the gonads and of the Müllerian ducts in each fetus. Fetus $L$ was an exception since it had small gonads (Plate 2 ) and relatively less inhibited Müllerian ducts: it belonged to a group of triplets with freemartin $\mathrm{K}$ which had larger gonads and larger ducts. The correlation still existed within the litter. When dealing with individual cases, it should be remembered that variations also exist in the degree of reduction of the Müllerian ducts in males.

(4) In order to verify the correlation between ovarian and Müllerian inhibition, the individual deviations from the theoretical curves were calculated ( $\Delta \mathrm{Y}$ for ovarian volume and $\Delta \mathrm{y}$ for Müllerian diameter), and plotted in Textfig. 6. There was a highly significant correlation between the degree of inhibition of gonads and Müllerian ducts $(r=0.75, P<0.001$ for 14 degrees of 
freedom), and a straight regression line could be calculated $(\Delta y=-0 \cdot 071+$ $20 \cdot 083 \Delta \mathrm{Y})$.

A correlation between the individual values of the ovarian volume $(\mathrm{Y})$ and of the diameter of the Müllerian ducts $(y)$ could also be calculated, but the

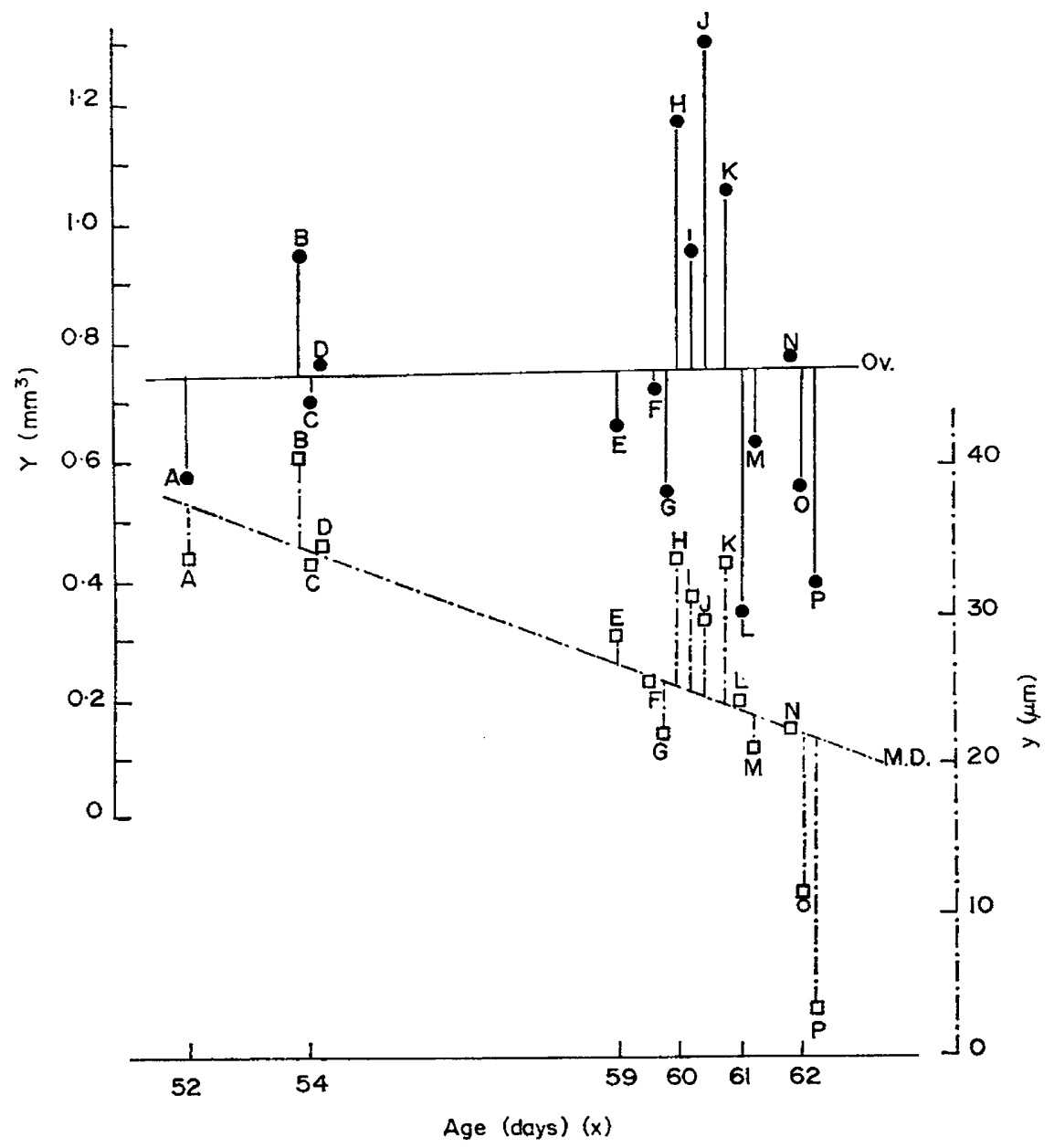

TEXT-FIG. 5. Correlation between gonadal and Müllerian inhibition in sixteen freemartins (A to P), 52 to 62 days old, in which both gonads and both Müllerian ducts were studied histologically. Ov. = calculated regression line for ovarian volume. M.D. = calculated regression line for Müllerian diameter. Individual values and deviations from the regression lines are shown by the vertical lines.

correlation coefficient was slightly less significant $(\mathrm{r}=+0.58, P=0.02$ for 14 degrees of freedom), because the influence of the age on the Müllerian diameter was neglected.

(5) In order to take into account those fetuses in which only the left Müllerian ducts had been studied, the individual deviations from the calculated curves for the left side were also compared for the whole group of twenty-six freemartins (A to $\mathrm{Z}$ ) aged 52 to 62 days. There was a highly significant correlation between 
the degree of inhibition of the left ovary and of the left Müllerian duct $(\mathrm{r}=$ $+0.60, P<0.001$ for 24 degrees of freedom). A highly significant correlation was also found to exist between the right ovary and the right Müllerian duct in the preceding group of sixteen fetuses $(\mathrm{r}=+0.79, P<0.001$ for 14 degrees of freedom).

\section{Masculine characters in freemartins}

The occurrence of masculine characters is not frequent in freemartins up to the age of 62 days. This subject will be discussed in the chronological sequence of appearance of these characters in normal males.

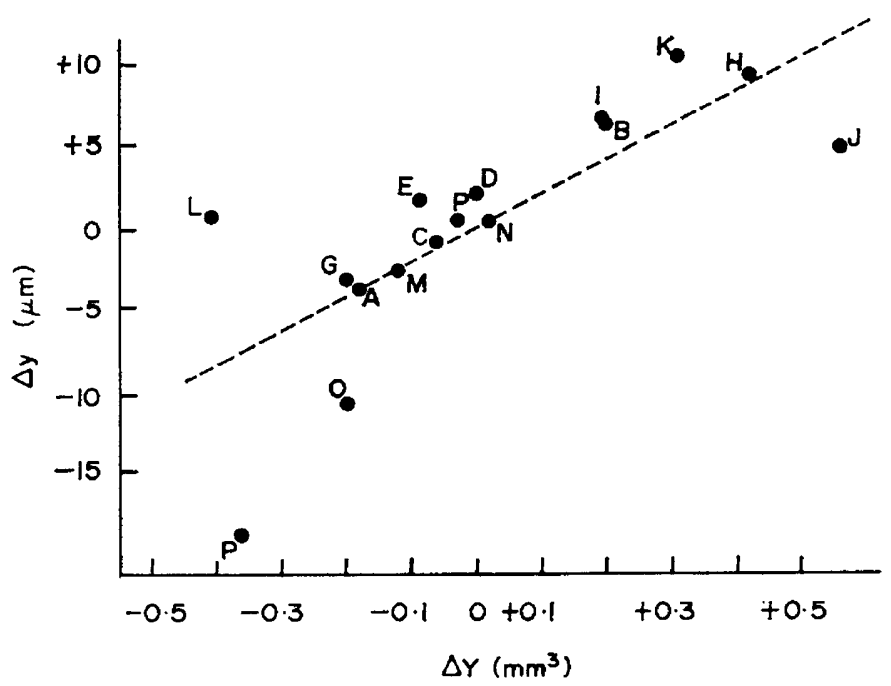

TEXT-FIG. 6. Correlation between individual deviations of ovarian volume $(\Delta Y)$ and Müllerian diameter $(\Delta y)$ from the corresponding calculated regression lines (see Textfig. 5).

External genitalia and ano-genital distance. In normal males, the penis migrates abdominally along the belly wall towards the umbilicus. The male ano-genital distance definitely increases from Day 47 until the penis comes to open below the umbilicus on Day 58. This sex character is very conspicuous during the developmental period extending up to Day 62 (Plate 2).

A possible indication of slight masculinization was found in a single 60-dayold freemartin ( $\mathrm{C} 43 \mathrm{~F} 5=\mathrm{Y}$, Tables 3,7 and 8) belonging to a group of sextuplets $(2 \hat{\sigma}+4$ \% $)$. The ovaries and the Müllerian ducts of the four females

\footnotetext{
Notes to Table 8:

The importance of vascular connections between fetuses is indicated schematically: heavy connections are schematized by a double junction line; anastomoses between fine vessels are indicated by a single junction line.

The degree of inhibition of the ovaries and Müllerian ducts was evaluated for each fetus by comparing the deviation of the individual value from the calculated regression line, as in Text-fig. 5.

For those fetuses in which only the left ovary and Müllerian ducts were measured, individual values were compared to regression lines calculated for the left ovaries and ducts.

+ , degree of inhibition less than calculated regression line; ++ , degree of inhibition equal to calculated regression line; +++ and ++++ , degree of inhibition superior to calculated regression line.
} 
TAble 8

INFLUENCE OF NUMBER, RELATIVE POSITION, AND VASCULAR CONNECTIONS OF FETUSES ON OVARIAN AND MÜLLERIAN INHIBITION IN FREEMARTINS

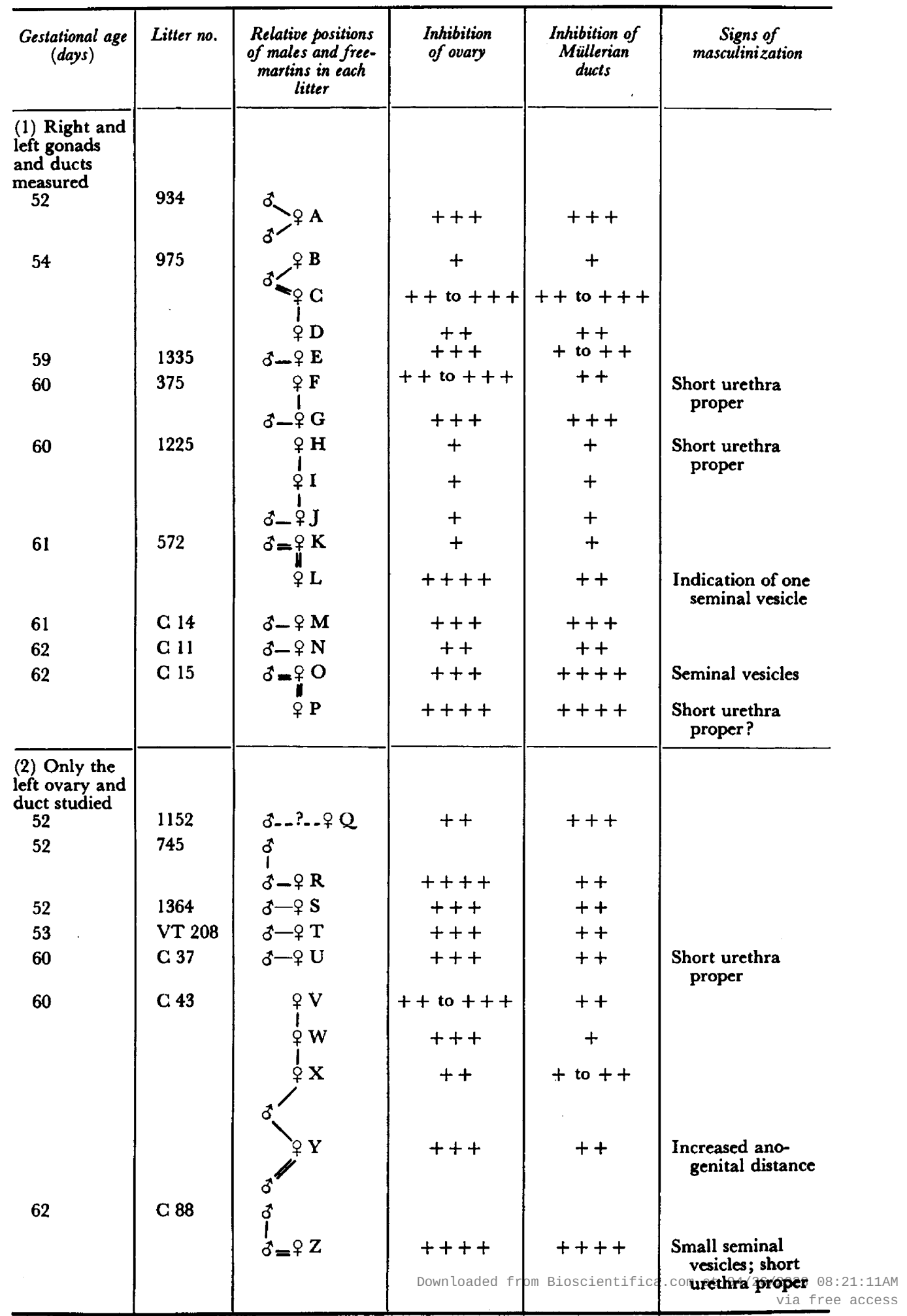


were strongly inhibited (Table 7); freemartin $\mathrm{Y}$ was located between two males and had a slightly increased ano-genital distance $(3.5 \mathrm{~mm}$ compared to $<2 \mathrm{~mm}$ in females and about $15 \mathrm{~mm}$ in males). The 'urethra proper' of this fetus was somewhat short, a masculine trend, but neither seminal vesicles of the male type nor a prostate were present.

In the three fetuses which had some development of seminal vesicles (C 15 $\mathrm{F} 2=\mathrm{O}, \mathrm{C} 88 \mathrm{~F} 1=\mathrm{Z}$ and $572 \mathrm{~F} 3=\mathrm{L}$ ) or in other fetuses in which the ovaries and Müllerian ducts were profoundly inhibited, no sign of masculinization of the external genitalia was noticed $(375 \mathrm{~F} 2=\mathrm{G}, \mathrm{C} 14 \mathrm{~F} 1=\mathrm{M}, \mathrm{C} 15 \mathrm{~F} 3=\mathrm{P}$, Tables 7 and 8, Text-fig. 5).

In normal males, the scrotum develops after Day 58 from paired genital swellings which at first are present in both sexes (Plate 2), but subsequently disappear in females (Bissonnette, 1924). On Day 60, the scrotum is an easily recognizable structure in males. No structure similar to a scrotum was found in any of our freemartins.

Male urogenital sinus and prostate. Differences correlated with the development of the prostatic urethra and the retention of early connections between urethra and Wolffian ducts in males, and with the development of the vagina and vestibule in females appear approximately on Day 56. They are far from being completely established on Day 62, the oldest stage studied in our freemartins.

(a) Upper urethra and prostatic urethra. After Day 55, the segment of the urethra situated between the bladder and the opening of the Wolffian ducts (upper urethra or 'urethra proper' of Bissonnette, 1924) remains shorter and broader in males, and consequently the prostatic urethra (posterior to the Wolffian opening) is longer. In females, the upper urethra increases rapidly in length in parallel with the development of the vagina, and consequently the posterior urethra becomes a relatively short vestibulum. Between Days 55 and 70 , the length of the 'urethra proper' (measured between the opening of the ureters in the bladder and the opening of the Wolffian ducts in the urethra) is increasing very rapidly in both sexes, but far more in females than in males. In females, its rate of increase is very high when related to increase in body length. Therefore, comparisons between freemartins and controls were made on the basis of the body length rather than age in days. Out of twelve freemartins aged 59 to 62 days, seven fetuses (G, I, J, K, L, M, O, Tables 7 and 8) were in the female range whereas four fetuses $(F, H, U, Z$ and perhaps $P$ ) were at least at the lower edge of the feminine values, and probably were intermediary between males and females. Some masculinizing influence would appear to be functioning in these fetuses, but there is no correlation between the degree of inhibition of the ovaries and of the Müllerian ducts and the probable reduction in length of the urethra proper. This sex character becomes more clearly defined after Day 70. Hence, the uncertainties of our conclusions.

(b) Prostate. In males, a few prostatic buds begin to grow on Day 56 on the prostatic urethra, which extends behind the opening of the Wolffian ducts. The number and the size of these buds increase slowly thereafter. No prostatic bud was ever observed in any freemartin of our series.

(c) Opening of the Wolffian ducts in the urogenital sinus. Sexual differences occur after Day 56 at the junction of the Wolffian ducts with the urogenital sinus, in 
relation to vaginal organogenesis in females (Bissonnette, 1924). These differences become more conspicuous as the fetus grows older. All our freemartins developed as females.

Seminal vesicles. The seminal vesicles appear in males on Day 56 as a slender outgrowth of the lower Wolffian duct which grows upwards. In females, the anterior Wolffian ducts regress slowly after Day 70, whereas their posterior part persists for a prolonged period. According to Bissonnette (1924), structures homologous to seminal vesicles appear in $7 \cdot 5-\mathrm{cm}$ long female fetuses.

No primordium of seminal vesicles was found in eleven freemartins on Days 59 and 60, whatever the degree of ovarian and Müllerian inhibition. The formation of seminal vesicles appeared in three out of seven freemartins on Days 61 and 62. Anlagen, similar to the early male seminal vesicles, were present in two cases, one on Day 61 (G 15 F2 = O, Tables 7 and 8, Text-fig. 5) and one on Day $62(\mathrm{C} 88 \mathrm{~F} 1=\mathrm{Z})$. A small unilateral outgrowth of the lower Wolffian duct seen in another freemartin (572 F3 = L, Tables 7 and 8, Textfig. 5) was probably a developing seminal vesicle. These three freemartins had severely inhibited ovaries, but it is noteworthy that: (1) the littermate freemartin $(P)$ of the first one had no seminal vesicles although its ovaries and Müllerian ducts were even more inhibited; (2) in Fetus $\mathbf{L}$ possessing an indication of a seminal vesicle, the Müllerian ducts were much less inhibited than in Fetus $\mathrm{P}$ which had no seminal vesicles, and the urethra was of a female type.

It would appear that a delayed development of seminal vesicles of the male type may occur on Days 61 or 62 in freemartins in which the ovaries are strongly inhibited, though there is no necessary link between ovarian or Müllerian inhibition and development of seminal vesicles. It is possible that seminal vesicles may still develop later, since anlagen homologous to seminal vesicles exist in females for a prolonged period. In older freemartins, they are commonly present though often poorly developed (Lillie, 1923; Bissonnette, 1924).

\section{Number and location of males in sets of multiple twins}

In multiple pregnancies, it is uncertain whether the intensity of the freemartin effect is associated with the relative number of males and females in a multiple set, the size of the vascular anastomoses or the possibility that the females located nearest to the male fetuses are more transformed than those which are separated from the male by another female. Table 8 shows the number and relative position of the males and females as well as the type of vascular connections. The last point is imprecise since it is based only on gross inspection of the vessels at dissection (see Plate 1). It should also be noted that in triplets, for instance, the possibility cannot be excluded that superficial connections on the chorion permit direct exchange of blood between Fetuses 1 and 3, by-passing the circulatory system of Fetus 2.

Relative number of males. Two males and one female: three cases (two on Day 52 and one on Day 62). In all three, the ovaries were very reduced in size. The 62day-old freemartin was among those showing the most pronounced ovarian and Müllerian inhibition. Two small seminal vesicles of the male type were present. 
One male and one female: seven cases $(52,52,53,59,60,61$ and 62 days of age). In six pairs, the vascular connections were slender and in one they were not studied (No. 1152, 52 days old). None of these fetuses was among the most severely modified freemartins; the degree of inhibition of the ovaries and of the Müllerian ducts varied from medium to strong.

Two males and four females: one case (60 days of age). Ovarian inhibition was medium to strong while Müllerian inhibition tended to be somewhat less severe.

One male and two females: three cases $(60,61$ and 62 days of age). Two freemartins had extremely inhibited ovaries ( $L$ and $P$ ); primordia of seminal vesicles were present in one of them $(L)$ and in another freemartin $(O)$. One fetus $(\mathbf{K})$ was much less affected than its sibling $(\mathbf{L})$.

One male and three females: two cases (54 and 60 days of age). The freemartin effect was rather feeble in one set (60 days) in which the vascular connections were slender. One of the three females of the other set was also less affected than its siblings; it had slender vascular connections with the male.

In conclusion, it seems difficult to correlate the freemartin effect with the relative number of males. In three groups of triplets in which two males were associated with one female, the female was severely affected; but the same degree of severity was observed in at least one female of each of two other 61and 62-day-old groups of triplets comprising two females and only one male. In the two groups of quadruplets comprising one male and three females, most of the females were mildly affected.

Relative situation of males and females. In some instances, the female located next to a male (No. 375, 60 days old), or between two males (No. C 43, 60 days old) was somewhat more transformed than the other females in the same set. However, the reverse was observed in other groups: in a group of 61-dayold triplets (No. 572), the female nearest to the single male was definitely less affected than the most distant female. In another group of triplets $(\mathrm{C} 15,62$ days old), the two females were nearly equally affected.

Size of blood connections. Although visual inspection of the vascular connections between fetuses gives only incomplete information, a few cases suggest that there might be a relation between the size of the vessels and the alterations in the females.

In the 54-day-old set of quadruplets (No. 975), the two females nearest to the male were unequally affected; this difference was paralleled by the size of the vascular connections. The three freemartins in which seminal vesicles were growing had well-developed vascular connections with their male co-twin.

Our observations do not show conclusively that a large number of males, or large vascular connections, favour the freemartin effect, although several cases suggest such a possibility.

\section{DISCUSSION}

\section{Ovary and upper Müllerian ducts in normal fetuses}

The growth of normal ovaries closely follows growth of the testes during the period studied in this paper, despite the fact that the testes are already mor- 
phologically differentiated, whereas ovarian organogenesis occurs later: premeiosis begins by Day 75 and inclusion of the oocytes into primordial follicles by Day 130, according to Erickson (1966).

The regression of the Müllerian ducts in males begins about Day 50. This appears first as a steady decrease in diameter, before progressive interruption and disappearance after Day 58.

In normal females, the diameter of the upper Müllerian ducts undergoes some decrease at the stage when they begin to retrogress in males. This reduction lasts for only a few days and is followed by an increase in diameter. The transitory reduction might result from several causes: (1) reorganization of the epithelial cells of the wall of the duct, accompanied by a thickening of the epithelium; (2) stretching of the duct if growth of the fetus exceeded elongation of the duct; (3) transitory presence in the normal female fetus of the Müllerianinhibiting substance which functions in males; against this is the fact that, in males, the decrease in diameter affects the most anterior level of the ducts whereas this part is little changed in females. The histological evidence would favour the first of these three possibilities (Plate 4), but no definitive statement can be made.

\section{Hormonal factors in the sex differentiation of the calf fetus}

There is no proof that the initial testicular differentiation in the calf fetus is imposed upon the gonadal primordium by any hormone or diffusible substance. Androgens given to the pregnant cow do not influence gonadal sex differentiation (Jost, Chodkiewicz \& Mauléon, 1963; Jost, 1965, 1966; Jainudeen \& Hafez, 1965). Similarly, there is no direct proof that organogenesis of the genital tract obeys a hormonal control in the calf fetus as it does in the rabbit fetus (Jost, 1947, 1953), though this seems probable. The production of biologically active androgens (Womak \& Koch, 1932; Atsumi, 1939) or of identified steroids (Weniger, Ehrhardt \& Fritig, 1967; Struck, Karg \& Jork, 1968) by calf testes after differentiation of the male genital tract has been reported.

Indirect indications contribute additional information. In preliminary experiments in vitro, the testes of a 59-day-old calf fetus inhibited the Müllerian ducts in pieces of the genital tract taken from 15-day-old rat fetuses; conversely, the Müllerian duct of a 54-day-old calf fetus was inhibited by testes of 15-day-old rat fetuses (Jost, Vigier \& Prepin, unpublished data). On the other hand, complete masculinization of the genital tract and external genitalia, in addition to preserved tubes, uteri and ovaries, was produced in female fetuses of cows given testosterone, methyltestosterone and $9 \alpha$-fluoro-methyltestosterone (Jost et al., 1963; Jost, 1965, 1966). The androgens masculinized the genital tract but failed to inhibit the Müllerian ducts. A similar condition of the genital tract (excepting for the presence of the testes) was observed in a 3-month-old male pseudohermaphrodite bull (Jost, 1965) in which tubes and well-developed uterine horns were present in addition to all masculine structures, including the penis. Such cases are also well documented in humans. These observations would suggest that discrete hormones inhibit the Müllerian ducts and stimulate the male characters in the calf fetus as well as in other species (Jost, 1965). If such were the case in the calf fetus, different substances would masculinize the 
external genitalia and inhibit the Müllerian ducts although these events occur almost simultaneously during normal development.

There is no proof so far that the fetal masculinizing hormone is testosterone and it may be noted that the antiandrogen cyproterone acetate given to the pregnant cow did not prevent masculinization of one male fetus (Jost, 1967). Variations in the hormonal production of calf testes are recognized. The prepubertal testis of the bull (Lindner, 1961) predominantly produces androstenedione while testosterone is the major post-pubertal and late fetal androgen (Struck et al., 1968), but nothing is known about the hormone controlling the early differentiation of the masculine tract of the calf.

\section{The early freemartin effect}

It is obvious that during the first week following the differentiation of the testes in males, i.e. after Day 40, the presumptive freemartins do not differ significantly from normal females (Plates 3 and 4). During the next 2 weeks, the freemartin effect becomes recognizable. At first, it consists only of an inhibition of the presumptive ovaries and of the Müllerian ducts but no masculinization of other sex characters occurs until Day 60. In normal males, the external genitalia are clearly masculinized from Day 47 , and other male characters appear after Day 56 (scrotum, prostate, seminal vesicles, etc.; Table 1).

The pioneers in the study of freemartins also stressed these early inhibitory effects. Keller \& Tandler (1916) described the effect of the male hormone as a 'Hemmungsreiz auf das in Bildung begriffene weibliche Genitale'. Lillie (1917) in the description of a 7.5-cm freemartin (probably 62 days old) emphasized, 'that the primary effect of the male sex hormone is to prevent the formation of the ingrowths that normally form the cortex of the ovary'. Bissonnette (1928a, b) also insisted upon the early inhibitory activity of the male hormone on the development of the 'secondary sex cords' in the ovary and on the Müllerian ducts. Both these authors noted that the development of masculine characters in freemartins-when present-was a later event. Our material confirms this view, though the upper limit (Day 62) of the developmental period covered by the present study does not permit an assessment of later events. In our series of freemartins, a very slight degree of masculinization of the external genitalia was probably present in only one freemartin out of thirtyeight individuals over 47 days. A scrotum or prostatic buds were never observed in eighteen freemartins over 56 days old; they are exceptional in fully grown freemartins. Seminal vesicles which are frequent in older freemartins (Lillie, 1923) were reported not to develop as early in freemartins as in males by Bissonnette (1924) who states 'the freemartin Wolffian duct derivatives show progressive modification of a female early differentiation toward a male later differentiation'. Small seminal vesicles or their presumptive anlagen were present in three out of seven freemartins on Days 61 and 62, though they were absent in the eleven freemartins studied between Days 56 and 60. This would agree with Bissonnette's views concerning these organs, but more individuals over 60 days of age should be studied. Structures homologous to seminal vesicles are still present in $20-\mathrm{cm}$ long normal females (Bissonnette, 1924) and over a prolonged period can probably be stimulated to grow and to persist. 
One important point of disagreement between our observations and earlier assumptions must be emphasized. Lillie $(1917,1923)$ suggested that the range of variation within the freemartin series could be accounted for by variation in the time at which vascular interchange is established or testicular action begins. This suggestion was accepted by other students of freemartins (Chapin, 1917; Bissonnette, 1924). As far as the ovaries and the Müllerian ducts are concerned, our material indicates that all the freemartins begin to be affected at the same stage, but this might not hold for other structures such as the seminal vesicles.

Inhibition of the presumptive ovaries and of the Müllerian ducts begins practically simultaneously, and coincides in time with the onset of Müllerian regression in males. Any development of masculine structure seems to be somewhat delayed in freemartins when compared with the normal male. This aspect is of peculiar interest when the freemartin gonads are considered. The early freemartin gonads are characterized by an arrested growth of the outer layers and of the whole organ, and absence of differentiation of the seminiferous tubules (Plate 3 ). We did not observe seminiferous tubules in any of our cases (up to an age of 62 days). As far as we are aware, no seminiferous tubules have been reported in the literature in freemartins under $20 \mathrm{~cm}$ crown-rump length. They were absent in freemartins of the following sizes: $3.2 \mathrm{~cm}$ (Bissonnette, $1928 \mathrm{a}) ; 3.75 \mathrm{~cm}$ (Lillie, 1923); 6.75 to $7.5 \mathrm{~cm}$ (three cases), $9 \mathrm{~cm}$ (two cases), $11.75 \mathrm{~cm}, 12.5 \mathrm{~cm}, 16.3 \mathrm{~cm}$ and $17 \mathrm{~cm}$ (Chapin, 1917; Bissonnette, 1924, 1928b) and on Day 60 (Rajakoski \& Hafez, 1964). On the contrary, seminiferous tubules were inconstantly found in $20-$ to $28-\mathrm{cm}$ freemartins. They were absent in three 20 - to $22 \cdot 5-\mathrm{cm}$ and $28-\mathrm{cm}$ fetuses reported by Chapin (1917). Seminiferous tubules were probably present in a 20-cm (Bissonnette, 1924) and in a $21.5-\mathrm{cm}$ (Chapin, 1917) freemartin (the latter was not described with precision). Extremely well developed seminiferous tubules containing germ cells were present in the $25-\mathrm{cm}$ freemartin described by Hay (1950), and many seminiferous tubules were found just after birth (Ohno, Trujillo, Stenius, Christian \& Teplitz, 1962; Goodfellow et al., 1965), or a few weeks after birth (Chapin, 1917; Willier, 1921; Moore, Graham \& Barr, 1957), or in older freemartins (Fraser-Roberts \& Greenwood, 1928; Buyse, 1936; Short, Smith, Mann, Evans, Hallet, Fryer \& Hamerton, 1969).

It appears that morphological 'masculinization' of the freemartin gonadwhen present-does not occur during the early developmental stages but that seminiferous tubules develop during a later phase, some time after the $20-\mathrm{cm}$ stage. This stage is of special significance in normal females since it coincides with the maximal thickness of the ovarian cortex (Ohno \& Smith, 1964), with the highest number of germ cells (Erickson, 1966), and with the differentiation of primary follicles. The first primary follicles appear in the normal ovary at the 15-cm stage (Thomson, 1942; Sakai, 1955) or somewhat later (Hashimoto \& Eguchi, 1955; Gropp \& Ohno, 1966; Erickson, 1966). If one accepts the notion that an ovary is differentiated only when primordial follicles separated by a stroma appear, the data suggest that seminiferous tubules may develop in the freemartin gonad at around the time of ovarian differentiation in normal females. If this interpretation of the available data is 
correct, it would appear that the agent responsible for the early changes in the freemartin gonad is not masculinizing on morphological grounds and that the appearance of seminiferous tubules takes place during a developmental phase that has to be studied as such. During the completion of this study, eleven additional freemartin fetuses ranging from 154 to $390 \mathrm{~mm}$, i.e. approximately from 87 to 153 days, were collected at the slaughterhouse with the cooperation of J. P. Perchellet. Structures closely resembling embryonic seminiferous tubules were observed in the gonads of five of them, usually near the posterior aspect of the rete system (fetuses of 175, 223, 254, 292 and $376 \mathrm{~mm}$ ). These observations support our previous interpretation.

Several factors of the intensity of the freemartin effect have been discussed in the past. Lillie (1923) showed that the vascular connections between twins may be established either through very large or through slender and delicate blood vessels, but he believed that 'the absence of correlation between the size of the vascular anastomosis and the degree of intersexuality, indicates an "all-or-none" type of reaction in formation of the freemartin'. The relative number of males and females in a multiple set has also been discussed. One male can modify two females in triplets (Bissonnette, 1928b), or four females in quintuplets (Rajakoski \& Hafez, 1963), but, in the latter, the effect was mild. Conversely, in a group of triplets, two males did not transform the female more than in a simple twin pregnancy (Bissonnette, 1928b). On the other hand, Buyse (1936) attributed the extreme modification he found in a $2 \frac{1}{2}$-year-old freemartin to the fact that it developed with two males in a group of triplets. Although we studied a larger number of animals, our evidence concerning the early freemartin effect was not very conclusive, because important differences were observed between different groups. The macroscopic examination of the vascular anastomoses cannot really reveal the volume of blood exchanged between fetuses, a factor which should be important if hormones are to be exchanged. We have also shown that there is no correlation between the proportion of XY cells present in the liver of fifteen of our freemartins and the severity of the freemartin effect (Vigier, Prépin \& Jost, 1972).

Moreover, it is not certain that the early inhibitory effects and the later masculinization of the seminal vesicles, epididymides and gonads depend on the same factor and can be studied together as one common freemartin effect.

The male co-twins to freemartins did not display any morphological abnormality up to Day 62. Recent evidence indicates that, in adulthood, these males have lower testosterone secretion rates than single born animals (Short et al., 1969; Pierrepoint et al., 1969). The cause of this difference and the period of life when it becomes established is still unknown.

\section{Interpretation of the early freemartin effects}

There is no doubt that the freemartin effect results from vascular connections between a male and a female fetus, and from exchange of blood, but the causative agent remains unknown. In the opinion of Lillie $(1917,1923)$, the fetal testicular hormone is transferred from the male to the female, although its exact mode of action in the female remains to be ascertained. He considered the theoretical possibilities that the testicular hormone could either be responsible 
for all the changes occurring at the level of the genital tract of the freemartin or only for the ovarian alterations which, in turn, impose alterations upon the other parts of the genital tract (Lillie, 1917). Witschi $(1939,1965)$ developed the latter view and assumed that only the testicular inductor 'medullarine' is transferred to the female partner. The freemartin ovaries are held to be responsible for the anomalies of the genital tract, and Witschi (1939) emphasized the parallel between the degree of the testicular character of the gonads and the degree of masculinization of the genital tract. Similarly, Short et al. (1969) and Short (1970), who recently observed that the gonads of an 11-month-old freemartin heifer secreted testosterone, believe that, "the varying degrees of masculinization of the freemartin's reproductive tract may well be due to the androgens secreted by its own gonads, and not to androgens from the fetal testis of the male co-twin' and 'favour the view that the female gonad is sex reversed by a humoral inductor substance coming from the male co-twin that causes retention of the medullary sex cords'. This last statement has to be amended since medullary sex cords cannot be recognized either in the young normal presumptive ovary or in the young freemartin gonad. It is also noteworthy that Leydig cells cannot be recognized at early stages.

Jost $(1966,1970)$ observed that the chronology of the early developmental history of the freemartins favours the possibility that a male inhibiting substance' inhibits both the presumptive ovaries and the Müllerian ducts.

Moore (1941, 1947) doubted the hormonal interpretation of the freemartin effect and conceived that it is 'due to humoral substances contributed by the male partner' but that these substances are 'produced in the entire organism and not by the gonads'. Possible immunological processes interfering with sex differentiation so far remain conjectural. When $\mathrm{XX} / \mathrm{XY}$ chimaerism in freemartin tissues was discovered, it was suggested that transfer of cells, rather than transfer of a hormone, was responsible for the freemartin effect (Fechheimer et al., 1963; Goodfellow et al., 1965; Kanagawa et al., 1965; Herschler \& Fechheimer, 1967). The authors of this concept did not discuss in detail which cells were concerned nor how they could achieve the freemartin condition. It might be speculated that the male cells modify only the freemartin gonad (which in turn would control the genital tract) or produce hormones inside the freemartin body. Whatever the agent which is transferred from the male to the female fetus, a consideration of some hormonal or humoral implications is not out of place. At least three different hypotheses can be considered.

(1) A single testicular hormone is responsible for all the changes observed in the freemartin. This hypothesis would not explain the lack of effect of the supposed male hormone on the external genitalia of the freemartin, unless these structures had a much higher threshold of sensitivity to this hormone than do the ovaries or the Müllerian ducts.

Moreover, if a single fetal testicular hormone were concerned in the freemartin effect, it is worth remembering that testosterone itself and its related compounds cannot duplicate the effects of the 'hormone' (Jost et al., 1963; Jost, 1965).

(2) The male factor is a testicular inductor (or imported cells) which acts only upon the presumptive ovaries of the freemartin. The ovaries become more 
or less sex reversed and impose modifications on the genital tract. The correlation between the degree of inhibition of the Müllerian ducts and of the ovaries reported here could be viewed as an argument in favour of the theory. From the same theory, however, the gonadal changes might be expected to precede those affecting the genital tract, whereas ovarian and Müllerian inhibition begin almost simultaneously, and coincide with Müllerian regression in the male.

The absence of masculinization of the phallus, scrotum, prostatic region, etc., in freemartins could also be accounted for if it resulted from a spatially limited secretory capacity of the freemartin gonads. There is an experimental model for such limited effects: in decapitated rabbit fetuses, testicular activity is decreased and the external genitalia fail to be masculinized whereas the internal structures are more masculinized the closer they are to the testes (Jost, 1948, 1951).

In evaluating the hypothesis that the freemartin effect results from an initial sex reversal of the presumptive ovaries, it should be recalled that the first freemartin effect seen in the presumptive ovaries is growth inhibition with no obvious trend to sex reversal. Morphological masculinization, i.e. differentiation of seminiferous tubules or of Leydig cells, is absent during the period of early sexual organogenesis in freemartins. It probably occurs long after the establishment of many masculine characters in males. It is impossible to state whether a physiological or endocrine masculinization of the freemartin gonads precedes any morphological masculinization, but the histological picture of the early freemartin gonads does not suggest intense secretory activity.

(3) The simultaneous inhibition of the presumptive ovaries in the freemartin and of the Müllerian ducts in both the male and the freemartin could be produced by a testicular 'antifeminine' substance or hormone, inhibiting both the ovary and the Müllerian ducts. This would easily account for the correlation between the ovarian and the Müllerian inhibition. This would also explain the simultaneous onset of both effects. The stimulation of male characters (in males or freemartins) would result from the production of another testicular masculinizing hormone. The condition of the developing freemartins necessitates the assumption that the masculinizing hormone does not easily reach them during the early stages. After Day 60 or so, increasing amounts of the masculinizing hormone might stimulate the seminal vesicles or other structures to the extent of response of which they are still capable. The site of production of the hormone remains unknown. It might originate in the male partner during the early stages and later be produced by the freemartin gonad itself.

The hypothesis of the dual nature of the early fetal testicular secretion calls for some comment. The 'antifeminine' factor, if normally secreted by the fetal testis, might play a double rôle in the male, both favouring the development of the tunica albuginea and inhibiting the Müllerian ducts. Its absence in some human male pseudohermaphrodites might explain the association of the persistence of Müllerian derivatives and of an abnormal tunica albuginea and outer testicular layer as described by Bergada, Cleveland, Jones \& Wilkins (1962) and by Jirasek (1970). The testicular hormone which, in the male calf fetus, masculinizes the external genitalia and the scrotum during the 2nd week after the differentiation of the testes, migrates relatively long distances, but 
is apparently not transferred to the presumptive freemartin. It might be distributed by preferential channels along the male genital system or inactivated before reaching the end organs of the co-twin. In connection with the former possibility, it has been reported that testes taken from 42- to 58-day-old calf fetuses and grafted under the skin of 18- to 20-day-old female rabbit fetuses did not masculinize their genital tracts (Jost, 1966).

At present, there is no proof that the hormone responsible for the early masculinization of the male genital tract in the calf fetus is testosterone.

\section{ACKNOWLEDGMENTS}

The cows used in this study were maintained at the Station de Physiologie Animale I.N.R.A., Jouy en Josas. We would like to thank: Professor Ch. Thibault, Dr R. Ortavant, Dr P. Mauléon and Dr F. du Mesnil du Buisson for their invaluable and friendly help and advice; Mr Y. De Fontaubert and Mr F. De La Chevalerie for their technical assistance, and Mr R. Scandolo for many photographs. Mr Y. Chirol, statistical engineer at the C.N.R.S., advised us on statistical matters. Mrs D. Touraud prepared most of the histological sections. The study was supported by a special grant from the Délégation Générale à la Recherche Scientifique et Technique.

\section{REFERENCES}

Atsum, Y. (1939) Research on the secretion of sex hormone during the embryonic stage. I. Male sex hormone during embryonic stage. F. Chosen med. Ass. 29, 99.

Bergada, C., Clevveland, W. W., Jones, H. W. \& Wirkins, L. (1962) Gonadal histology in patients with male pseudohermaphroditism and atypical gonadal dysgenesis: relation to theories of sex differentiation. Acta endocr., Copenh. 40, 493.

BissonNETTE, T. H. (1924) The development of the reproductive ducts and canals in the freemartin with comparison of the normal. Am. F. Anat. 33, 267.

BissonNetTe, T. H. (1928a) Notes on a 32 millimeter freemartin. Biol. Bull. mar. biol. Lab., Woods Hole, $54,238$.

BissonNETTE, T. H. (1928b) Notes on multiple pregnancies in cattle with special reference to three cases of prenatal triplets and the freemartins involved. Am. J. Anat. 42, 29.

BuRNs, R. K. (1961) Role of hormones in the differentiation of sex. In: Sex and Internal Secretions, Vol. 1, Ch. 2, p. 76. 3rd edn. Eds. W. G. Young and G. W. Gorner. Williams \& Wilkins, Baltimore.

Buyse, H. (1936) A case of extreme sex modification in an adult bovine freemartin. Anat. Rec. 66, 43.

Chapin, C. L. (1917) A microscopic study of the reproductive system of fetal freemartins. F. exp. Zool. 23, 453.

ERICkson, B. H. (1966) Development and radio-response of the prenatal bovine ovary. F. Reprod. Fert. $11,97$.

Fechuemer, N. S., Herschler, M. S. \& Gilmore, L. O. (1963) Sex chromosome mosaicism in unlike sexed cattle twins. In: Genetics Today, p. 265. Pergamon Press, London.

Fraser-Roberts, J. A. \& Greenwood, A. W. (1928) An extreme freemartin and freemartin-like condition in sheep. 7. Anat. 63,87.

Goodfellow, S. A., Strong, S. J. \& STEwart, J. S. S. (1965) Bovine freemartins and true hermaphroditism. Lancet, i, 1040.

Gropp, A. \& OhNo, S. (1966) The presence of a common embryonic blastema for ovarian and testicular parenchymal (follicular, interstitial and tubular) cells in cattle, Bos taurus. Z. Zellforsch. mikrosk. Anat. 74, 505.

Hay, D. (1950) Etude d'un free-martin spontané chez les ruminants. Archs Anat. Histol. Embryol. 35, 55.

Hashimoto, Y. \& Eguchi, Y. (1955) Histological observations on the gonads in the cattle and the horse fetus. 1. The cattle fetus. Fap. F. zootech. Sci. 26, 259.

Herschler, H. S. \& FechHeImer, N. S. (1967) The role of sex chromosome chimerism in altering sexual development of mammals. Cytogenetics, 6, 204. 
Jainudeen, M. R. \& Hafez, E. S. E. (1965) Attempts to induce bovine freemartinism experimentally. 7. Reprod. Fert. 10, 281.

JIRASEK, J. E. (1970) The relationship between differentiation of the testicle, genital ducts and external genitalia in fetal and postnatal life. In: The Human Testis, p. 19. Eds. E. Rosemberg and G. A. Paulsen. Plenum Press, New York.

Jost, A. (1947) Recherches sur la différenciation sexuelle de l'embryon de lapin. III. Rôle des gonades foetales dans la différenciation sexuelle somatique. Archs Anat. microsc. Morph. exp. 36, 271.

Jost, A. (1948) Le contrôle hormonal de la différenciation du sexe. Biol. Rev. 23, 201.

Jost, A. (1951) Recherches sur la différenciation sexuelle de l'embryon de lapin. IV. Organogenèse sexuelle masculine aprés décapitation du foetus. Archs Anat. microsc. Morph. exp. 40, 247.

Jost, A. (1953) Problems of fetal endocrinology: the gonadal and hypophyseal hormones. Recent Progr. Horm. Res. 8, 379.

Jost, A. (1965) Gonadal hormones in the sex differentiation of the mammalian fetus. In: Organogenesis, p. 611 . Eds. R. L. de Haan and H. Ursprung. Holt, Rinehart \& Winston, New York.

Jost, A. (1966) Steroids and sex differentiation of the mammalian foetus. Excerpta Medica Int. Congr. Ser. $132,74$.

Jost, A. (1967) Modalities in the action of androgens on the foetus. Research on Steroids, 3, 207.

Jost, A. (1970) Hormonal factors in the sex differentiation of the mammalian foetus. Phil. Trans. R. Soc. Lond. B, 259, 119.

Jost, A., Chodkrewicz, M. \& Mauléon, P. (1963) Intersexualité du foetus de veau produite par des androgènes. Comparaison entre l'hormone foetale responsable de free-martinisme et l'hormone testiculaire adulte. C. r. hebd. Séanc. Acad. Sci., Paris, 256, 274.

Kanagawa, H., Kawata, K. \& Ishikawa, T. (1965) Chromosome studies on heterosexual twins in cattle. II. Significance of sex chromosome chimerism $(\mathrm{XX} / \mathrm{XY})$ in early diagnosis of freemartin. Jap. 7. vet. Res. 13, 33.

KelLeR, K. \& TANDLER, J. (1916) Über das Verhalten der Eihäute bei der Zwillingsträchtigkeit des Rindes. Untersuchungen über die Enstehungsursache der Geschlechtichen Unterentwicklung von weiblichen Zwillingskälbern welche neben einem mannlichen Kalbe zur Entwickelung gelangen. Wien. tierärtzl. Wschr. 3, 513.

KREhBiel, E. B. (1963) Differentiation of gonads in the bovine embryo. Dissertation Ph.D., Kansas State University, U.S.A.

LILLIE, F. R. (1916) Theory of the freemartin. Science, $\mathcal{N} . Y_{.}$43, 611.

LILLIE, F. R. (1917) The freemartin, a study of the action of sex hormones in foetal life of cattle. F. exp. Zool. 23, 371 .

LrLlie, F. R. (1923) Supplementary notes on twins in cattle. Biol. Bull. mar. biol. Lab., Woods Hole, 44, (2), 47.

LindNer, H. R. (1961) Androgens and related compounds in the spermatic vein blood of domestic animals. I. Neutral steroids secreted by the bull testis. F. Endocr. 23, 139.

Mauléon, P., Mariana, J. G., Benoit, M., Solari, A. \& Ghupin, D. (1970) Influence de différentes doses de P.M.s.g. et H.C.G. injectées en phase folliculaire du cycle oestrien, sur le nombre et le rendement d'ovulations de vaches de race Française Frisonne pie noire. Annls Biol. anim. Biochim. Biophys. 10, 31 .

MOore, G. R. (1941) On the role of sex hormones in sex differentiation in the opossum (Didelphys virginia). Physiol. Zoöl. 14, 1.

MOORE, C. R. (1947) Embryonic sex hormones and sexual differentiation. In: American Lecture Series. Charles C. Thomas, Springfield, Ill.

Moore, K. L., Graham, M. A. \& Barr, M. L. (1957) The sex chromatin of the bovine freemartin. F. exp. Zool. 135, 101.

Oнno, S. \& Sмттн, J. B. (1964) Role of fetal follicular cells in meiosis of mammalian oocytes. Cytogenetics, 3, 324.

Ohno, S., Trujillo, J., Stenius, C., Ghristian, L. C. \& Teplitz, R. (1962) Possible germ cell chimeras among newborn dizygotic twin calves (Bos taurus). Cytogenetics, 1, 258.

Pierrepoint, C. G., Stewart, J. S. S. \& Rack, J. (1969) Patterns of steroid biosynthesis in the gonads of freemartins and their male co-twins. F. Reprod. Fert. Suppl. 7, 63.

RajaKoski, E. \& HAFEz, E. S. E. (1963) Derivations of cortical cords in adult free-martin gonads of bovine quintuplets. Anat. Rec. 147, 457.

Rajakoski, E. \& Hafez, E. S. E. (1964) Cytological differentiation of fetal bovine gonads. Cytogenetics, $3,193$.

SAKAI, T. (1955) Studies on the development of the embryonic ovary in swine, cattle, and horse. $7 a p$. 7. vet. Res. 3, 183.

Short, R. V. (1970) The bovine free-martin: a new look at an old problem. Phil. Trans. R. Soc. B, 259,141 . 
Short, R. V., Smith, J., Mann, T., Evans, E. P., Hallett, J., Fryer, A. \& Hamerton, J. L. (1969) Cytogenetic and endocrine studies of a freemartin heifer and its bull co-twin. Cytogenetics, 8, 369.

Simpson, G. G., Roe, A. \& Lewontin, R. C. (1960) Quantitative zoology. Revised edn, p. 440. Harcourt, Brace \& Co, New York, Burlingame.

Struck, H., KARg, H. \& JoRK, H. (1968) Dünnschichtchromatographische Bestimmung von Testosteron und $\Delta_{4}$-Androstendion (3-17) aus Testesgewebe von Rinderföten. 7. Chromat. 36, 74.

Thompson, J. D. (1942) Comparative studies on gonad development in the rat, the pig and in cattle. Proc. Iowa Acad. Sci. 49, 475.

Vigier, B., Prépin, J. \& Jost, A. (1972) Absence de corrélation entre le chimérisme XX/XY dans le foie et les premiers signes de freemartinisme chez le foetus de veau. Cytogenetics, (In press).

Weniger, P., Ehrhardt, J. D. \& Fritug, B, (1967) Sécrétion de testostérone et d'androstènedione par les testicules foetaux de veau cultivés in vitro. C. r. hebd. Séanc. Acad. Sci., Paris, 264, 1911.

WILLIER, B. H. (1921) Structure and homologies of free-martin gonads. F. exp. Zool. 33, 63.

Willier, B. H. (1939) The embryonic development of sex. In: Sex and Internal Secretions, 2nd edn, Chap. 3, p. 64. Eds. E. Allen, C. Danforth and E. A. Doisy. Williams \& Wilkins, Baltimore.

Wrtschi, E. (1939) Modification of the development of sex in lower vertebrates and in mammals. In: Sex and Internal Secretions, 2nd edn, Vol. 3, p. 145. Eds. E. Allen, G. Danforth and E. A. Doisy. Williams \& Wilkins, Baltimore.

WrTschr, E. (1965) Hormones and embryonic induction. Archs Anat. microsc. Morph. exp. 54, 601.

Womak, E. B. \& Kock, F. C. (1932) Studies on extraction of testicular hormone from tissues and its quantitative distribution therein. Endocrinology, 16, 267. 